\title{
Efficient aeroelastic wing optimization through a compact aerofoil decomposition approach
}

\author{
Daniel J. Poole ${ }^{1}$ (Christian B. Allen ${ }^{1} \cdot$ Thomas C. S. Rendall $^{1}$
}

Received: 3 September 2021 / Revised: 7 January 2022 / Accepted: 10 January 2022 / Published online: 8 February 2022

(c) The Author(s) 2022

\begin{abstract}
Efficient optimization of an aeroelastic wing is presented through multi-disciplinary analysis using low-dimensional modal design variables. Much work in wing optimization has concentrated on high-fidelity surface control, therefore utilising often hundreds of design variables. However, whilst fine surface control can be useful, problems can arise such as large disparities in design variable values when planform variables are introduced, slow convergence speeds, and lack of compatibility with global algorithms. Therefore, the focus of this paper is to filter the design space of this problem to reduce the dimensionality and complexity of the problem. Orthogonal geometric design variables are derived in the geometric space via singular value decomposition. Orthogonality of design variables leads to a well-conditioned design space and ensures effective optimizer convergence. These variables are applied in a sectional fashion for fixed planform drag minimization of a flexible transonic wing, using a gradient-based optimizer. Shock-free solutions are demonstrated when optimizing a rigid wing, indicating suitability of the aerofoil modes for sectional-based wing optimization. However, it is shown that these wings have poor performance when subsequently deformed under flight loads, hence optimisation including full aeroelastic performance is performed. Encouragingly, shock-free solutions are again computed. Loading is shifted outboard, leading to increased tip deflection. Monotonic improvement in the objective function (drag) with increase in dimensionality is also proven. Furthermore, applying these sectional deformation modes globally across the wing with only 10 design variable leads to a $28 \%$ drag reduction, which is within 7 drag counts of when the modes are applied locally through 82 design variables. This therefore opens the possibility of introducing global optimization algorithms to high-fidelity aeroelastic wing optimization.
\end{abstract}

Keywords SVD $\cdot$ Decomposition $\cdot$ Optimization $\cdot$ Aeroelasticity

\section{Introduction}

The design of an aircraft is inherently an exercise in the coupling and subsequent compromise of multiple, and often independent, disciplines. Nowhere is this more apparent than in the design of aircraft wings, where the aerodynamic performance typically dominates, but where the design is dictated by the behaviour of many other disciplines. Multidisciplinary analysis (MDA) is the framework that proceeds when analysis of each discipline is integrated into an overall coupled system. Furthermore, beyond analysis, the overall

Responsible Editor: Axel Schumacher

Daniel J. Poole

d.j.poole@bristol.ac.uk

1 Department of Aerospace Engineering, University of Bristol, Bristol BS8 1TR, UK multi-disciplinary system may be linked to an optimization process leading to the multi-disciplinary optimization (MDO) framework (Martins and Lambe 2013).

Commonly, engineering optimization problems embed high-fidelity computational analysis into the process, which is used to determine various metrics against which to optimize. The problem then reduces to that of finding the optimum design (often a geometric problem) that optimizes a cost function subject to restrictions imposed by constraint functions. The cost and constraint functions typically rely on outputs from the analysis. An optimization algorithm is then required to link the analysis to the design process, where the algorithm uses values of the cost and constraints to determine a vector of design variables that link to changes in the design to hopefully improve the cost. A parameterization is the vehicle that governs how the design variables affect the design. An example single-discipline problem is finding the optimum aerodynamic aerofoil or wing shape to minimise 
drag subject to a constraint on lift, where a computational fluid dynamics (CFD) solver is used to determine the aerodynamic performance; see Hicks and Henne (1978) Qin et al. (2004), Morris et al. (2008), Allen and Rendall (2013), Poole et al. (2017, 2018b), Destarac et al. 2018) for example.

In aircraft wing design, the aero-structural-coupled system dominates the performance. When determining aerodynamic performance of the wing in flight, the rigid wing shape is deflected as aerodynamic loads are transferred to the structure and an equilibrium state results. Simulation of this system commonly requires coupling of separate CFD and computational structural mechanics (CSM) solvers in a partitioned manner (Geuzaine et al. 2003; Taylor et al. 2004; Woodgate et al. 2005; Kenway and Martins 2014). This solution approach has the advantage of requiring little development of new codes, with the main development requirements being the solution coupling, and the force and displacement interpolation and mesh deformation needed due to non-coincident fluid and structural meshes. The coupling requires that an outer iteration loop be added to march the solution to equilibrium. The interpolation and mesh deformation are often handled separately, but a unified approach using radial basis function (RBF) interpolation was shown to be highly effective (Rendall and Allen 2008). A similar approach, but using B-spline-based methods, has also recently been presented (Zhang et al. 2017).

An alternative solution approach is the monolithic method, where a single solver that solves the governing equations synchronously is developed (Michler et al. 2004; Hübner et al. 2004). Whilst accuracy and convergence tends to be better with the monolithic approach, the development costs are a barrier. For example, all four codes that submitted results to the aero-structural benchmarking case of the Sixth AIAA CFD Drag Prediction Workshop used a partitioned solution approach (Keye and Mavriplis 2018).

Once a suitable aero-structural solver is developed, full MDO of the wing that optimizes for the performance of the coupled aerodynamic and structural responses can be realised. There exists a deep history of performing ever increasing fidelity of aero-structural optimization (SobieszczanskiSobieski and Haftka 1997). Due to the cost associated with aero-structural optimization, local gradient-based optimization algorithms have usually been the tool of choice. The problem then exists of determining the gradients of the cost and constraints with respect to the design variables. The adoption of the adjoint approach (Jameson 1988) permits all gradients of flow quantities with respect to surface changes to be evaluated for a computing cost that is similar to a single flow solution. Using this, coupled aero-structural adjoint methods have been presented (Maute et al. 2001; Martins et al. 2005; Kenway et al. 2014), which has permitted highfidelity, large-scale aero-structural optimization. Furthermore, recent developments include the ongoing work to couple more disciplines into the process, including coupling aerodynamics, structures and acoustics in a fully coupled unsteady optimization of rotorcraft (Mavriplis et al. 2017).

Large-scale, high-fidelity MDO lends itself to detailed shape optimization. Such problems often have very large numbers of design variables. However, the quantity of design variables often has a significant effect on the convergence of the optimizer, as well as the definition of multimodality in the problem. Furthermore, whilst gradient-based methods are the most popular optimization algorithms in MDO, if alternatives (such as population-based) methods are to be exploited, then lower-dimensional design spaces become key, particularly as it been shown that the number of function evaluations increases quadratically for increasing numbers of design variables (Lyu et al. 2014). An alternative approach can also be to employ multi-start gradient (Chernukhin and Zingg 2013). Dimensionality reduction techniques have become a useful approach for reducing the dimensionality associated with aerodynamic shape optimization. Of these, singular value decomposition-based approaches take a training matrix of data (for example a number of aerofoil shapes (Toal et al. 2010; Ghoman et al. 2012)), and project a reduced-order basis approximation of the original data. Work by the authors has shown that this is a very efficient approach for producing a reduced set of aerofoil deformation modes (Poole et al. 2015a) that are suitable for aerodynamic optimization of aerofoils (Poole et al. 2017) and wings (Allen et al. 2018). Furthermore, comprehensive experiments (Masters et al. 2017) have shown singular value decomposition (SVD) modes to be the most efficient approach at representing a generic aerofoil compared to most other commonly used parameterization methods, and have shown to produce single figure drag values for the AIAA ADODG Test Case 1 (Kedward et al. 2020b), when combined with a surface gradient limiting constraint (Kedward et al. 2020a).

The work presented in this paper extends the application of these modes further by considering high-fidelity aerostructural wing optimization. The overall goal of this work is to make high-fidelity global multi-disciplinary optimization of wings a reality, and the use of a compact aerofoil decomposition for the design variables is a pillar to this goal. Whilst fixed planform optimization of rigid aerodynamic wings is generally considered to be unimodal ( $\mathrm{Yu}$ et al. 2018), introducing planform changes along with sectional changes introduces clear multimodality (Poole et al. 2018b; Streuber and Zingg 2021). Furthermore, similar behaviour is also likely to be seen in aeroelastic wing optimization so there is a clear need to consider how global methods could be introduced into high-fidelity wing optimization. Specifically, the objectives of this work are to first determine the suitability of the aerofoil decomposition design variables for aeroelastic optimization and ensure no adverse effects 
are found when structural responses are included in the problem. Second, this work aims to determine whether a compact wing deformation approach with only a handful of sectional variables, can be successful in high-fidelity elastic wing optimization.

The remainder of the paper is organised as follows: the compact modal decomposition and how this is applied to wing optimization is outlined in Sect. 2; the solver and datum solution is presented in Sect. 3; the optimization problem and chosen optimizer is presented in Sect. 4; optimization of the rigid wing and aeroelastic wing are presented in Sects. 5 and 6 , respectively; finally, conclusions are given in Sect. 7.

\section{Decomposition for wing design variables}

The design variables used in the optimization process are the weightings of various sectional deformations. These sectional deformations come about by performing a matrix decomposition that uses SVD on a training library of aerofoils. The resulting modes represent a reduced basis projection of the full-basis aerofoil design space. The modes are also orthogonal, which leads to a well conditioned design space, and no coupling of variables in the geometric space. This aids optimizer convergence considerably. The surface deformation modes are defined in the two-dimensional surface space, so need mapping onto both the three-dimensional surface space and the volume space. This is achieved in a unified manner through radial basis function (RBF) interpolation via a set of control points. As RBF interpolation only needs a point cloud and no connectivity, it is general. This also leads to aerofoil surface modes being general.

\subsection{SVD for sectional deformations}

To obtain aerofoil deformation modes, a training library of aerofoils needs to first be collated. The selection of the training library is one of the most important steps in this process since the characteristics of the library map to the characteristics of the deformation modes, therefore, careful selection of the training library is key to allowing a wide design space to be represented and therefore allowing a variety of aerofoil shapes to be explored. The authors have previously published work (Poole et al. 2015a) that used a metric-based filtering approach to select the training library. In this work, the library of aerofoils is as previously used by the authors for transonic aerofoil optimization (Poole et al. 2017). The aerofoil data was obtained from the UIUC database ${ }^{1}$, and

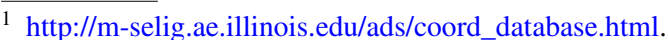

was subsequently smoothed and re-parameterised to ensure a consistent fractional arc-length distribution of points.

A training library contains $M$ aerofoils each parameterised with $N$ surface points, where the $i$-th surface point of the $m$-th aerofoil has coordinates $\left(x_{i_{m}}, z_{i_{m}}\right)$. To obtain aerofoil deformation modes, the vector difference between each surface point of all aerofoils is computed. The vector difference of the $i$-th surface point between the $m$-th and $n$-th aerofoils is given as $\left(\Delta x_{i_{m, n}}, \Delta z_{i_{m, n}}\right)$. It is worth noting that one option is to consider vertical deformations only (in this case, the aerofoils would need to have equal horizontal point distributions) but this can be restrictive in areas of high curvature, such as the leading edge so both horizontal and vertical deformations are considered. More detail is given in (Poole et al. 2015a).

All deformations are collated into a single deformation matrix that has $2 N$ rows and $M_{\mathrm{def}}=M(M-1) / 2$ columns:

$\boldsymbol{\Psi}=\left(\begin{array}{cccccc}\Delta x_{1_{1,2}} & \cdots & \Delta x_{1_{1, M}} & \Delta x_{1_{2,3}} & \cdots & \Delta x_{1_{M-1, M}} \\ \vdots & \ddots & \vdots & \vdots & \ddots & \vdots \\ \Delta x_{N_{1,2}} & \cdots & \Delta x_{N_{1, M}} & \Delta x_{N_{2,3}} & \cdots & \Delta x_{N_{M-1, M}} \\ \Delta z_{1_{1,2}} & \cdots & \Delta z_{1_{1, M}} & \Delta z_{1_{2,3}} & \cdots & \Delta z_{1_{M-1, M}} \\ \vdots & \ddots & \vdots & \vdots & \ddots & \vdots \\ \Delta z_{N_{1,2}} & \cdots & \Delta z_{1_{1, M}} & \Delta z_{N_{2,3}} & \cdots & \Delta z_{N_{M-1, M}}\end{array}\right)$.

The deformation matrix has an SVD given by:

$\boldsymbol{\Psi}=\mathbf{U} \boldsymbol{\Sigma} \mathbf{V}^{T}$

where $\mathbf{U}$ is an orthonormal $2 N \times 2 N$ matrix, $\boldsymbol{\Sigma}$ is a diagonal matrix with $\min \left\{2 N, M_{\text {def }}\right\}$ diagonal entries arranged in descending order, and $\mathbf{V}^{T}$ is an orthonormal $M_{\mathrm{def}} \times M_{\mathrm{def}}$ matrix. The columns of $\mathbf{U}$ contain the aerofoil deformation modes, and these are extracted column-wise and used for the optimization. The diagonal entries of $\boldsymbol{\Sigma}$ contain the singular values, which may be used to determine now much 'energy' each mode has in the overall system.

The use of the SVD here is for geometric filtration of the high-degree training matrix into a low-degree representation using deformation modes. To do this, the first $D$ modes (i.e. first $D$ columns of $\mathbf{U}$ ) may be extracted and a $D$-rank approximation of the original matrix may be obtained by:

$\boldsymbol{\Psi} \approx \Psi^{(D)}=\tilde{\mathbf{U}} \widetilde{\boldsymbol{\Sigma}} \tilde{\mathbf{V}}^{T}$

where the tilde is used to denote reduced forms of the SVD matrices. Once a low-rank approximation is found through SVD, the following is true (Eckart and Young 1936):

$\left\|\boldsymbol{\Psi}-\boldsymbol{\Psi}^{(D)}\right\|_{F} \leq\|\boldsymbol{\Psi}-\boldsymbol{\Phi}\|_{F}$,

where $\boldsymbol{\Phi}$ is any matrix of $\operatorname{rank} D$ and $\|\cdot\|_{F}$ is the Frobenius norm. Hence, the error between the low-rank approximation and the full rank approximation will always be at least as 


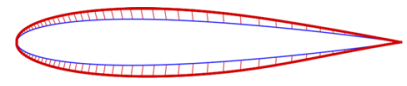

(a) Mode 1

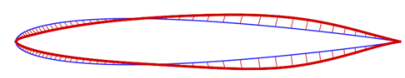

(c) Mode 3

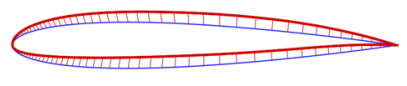

(b) Mode 2

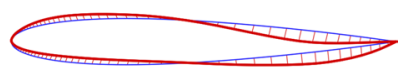

(d) Mode 4
Fig. 1 First four aerofoil deformation modes (superimposed on NACA0012 for visualisation)

good as the error between any other $k$-rank matrix and the full-rank matrix. In this sense, the SVD produces an optimal low order projection of the higher-dimensional space into the lower-dimensional one.

The first four deformation modes are shown in Fig. 1 (these are superimposed on NACA0012 for visualisation purposes). It is clear to see that the first mode represents a thickness change, then the second represents a camber change, indicating that for this library of aerofoils, these are the two most important aerofoil design parameters respectively.

Given the required number of deformation modes, $D$, in the optimization, the design variables are the weightings of each deformation mode. The overall deformation is then a linear superposition of each mode:

$\Delta \mathbf{X}=\sum_{i=1}^{D} \alpha_{i} \mathbf{U}_{i}$,

where $\alpha_{i}$ is the design variable relating to the $i$-th mode and $\mathbf{U}_{i}$ is the $i$-th mode, which is the $i$-th column of $\mathbf{U}$. Since the modes are independent of one another, the design space of lower-dimensional problems is entirely contained within the design space of higher dimensional problems. As such, adding further modes can only lead to at least a better optimum objective function. For aerofoil drag optimization, experience of the authors shows that there are normally few benefits of going above 10 modes, and that if there is an improvement in the objective function, this is outweighed by the additional cost due to increases in optimizer iterations and gradient evaluations. This is therefore an extremely efficient method of performing aerofoil optimization.

\subsection{Application to wing deformation}

The aerofoil deformation modes are surface deformations, however, to ensure body-fitted meshes are retained in the optimization the mesh also needs to deform. Furthermore, the surface deformation modes are applied sectionally, which is defined here.
The sectional deformations are applied at a fixed number of spanwise stations, i.e. Eq. 4 is applied at these stations locally. However, the sectional deformations can also be applied in a global nature. The sectional deformations are applied using the RBF control point approach, where a set of control points are defined in the fluid domain and global volume interpolation translates deformation of the control points to deformation of the aerodynamic mesh. Hence, the modal deformations are used to drive deformation of the control points that subsequently deform the wing surface and mesh. These deformations are decoupled, so the control point modal deformations are determined off-line and then applied in the optimization process. This has the added advantage of producing modal deformations that are not restricted to the original discretisation (i.e. number of points - rows of Eq. 1 - and distribution of points) used to derive them.

At the heart of this technique in an RBF interpolation developed originally for aero-structure coupling and mesh motion by Rendall and Allen (2008). An RBF interpolation, $s$, is a linear combination of basis functions, whose argument is the Euclidean distance, $\|\cdot\|$ between the point to be interpolated in the domain, $\mathbf{x}$, and the $N$ points in the known data set. Therefore, the influence that a known point has is controlled by a function, $\phi$, that depends on the distance from the interpolated point:

$s(\mathbf{x})=\sum_{i=1}^{N} \beta_{i} \phi\left(\left\|\mathbf{x}-\mathbf{x}_{i}\right\|\right)+p(\mathbf{x})$.

Control points decouple the shape deformations from the surface mesh and provide a unified framework for surface and mesh deformation. Given $n_{c}$ control points, a global $\mathrm{RBF}$ interpolation of this nature provides exact recovery of data at known sites, and interpolation of that data away from the sites. In the case of optimization, the data to be interpolated is deformation of the control points, hence a deformation field is created. The position of the aerodynamic mesh points in the field therefore defines the deformation of those points. Since exact recovery of data at the know sites (in this case the position of the control points, which for the $j$-th control point is defined as $\left.\left(x_{c_{j}}, y_{c_{j}}, z_{c_{j}}\right)\right)$ is specified, the interpolation takes the form:

$\Delta \mathbf{x}_{c}=\mathbf{M} \boldsymbol{\beta}^{x}$,

where $\Delta \mathbf{x}_{c}=\left[\Delta x_{c_{1}}, \ldots, \Delta x_{c_{n_{c}}}\right]^{T}, \boldsymbol{\beta}^{x}=\left[\beta_{1}^{x}, \ldots, \beta_{n_{c}}^{x}\right]^{T}$ and

$\mathbf{M}=\left(\begin{array}{ccc}\phi_{1,1} & \cdots & \phi_{1, n_{c}} \\ \vdots & \ddots & \vdots \\ \phi_{n_{c}, 1} & \cdots & \phi_{n_{c}, n_{c}}\end{array}\right)$.

Analogous definitions hold for the $y$ and $z$ coordinates. The radial basis function $\phi_{i, j}=\phi\left(\left\|\mathbf{x}_{c_{i}}-\mathbf{x}_{c_{j}}\right\|\right)$ can take a number of forms, but the radially decaying functions of Wendland 
(2005) are a good choice for the mesh deformation problem to give the interpolation a local character and ensure deformation is contained in a region near the moving body. The $C^{2}$ function is used here.

Once the linear system is solved, the resulting deformation field can be evaluated at the location of each mesh point. The deformation of an aerodynamic mesh point is given by:

$\Delta x_{a}=\sum_{i=1}^{n_{c}} \beta_{i}^{x} \phi\left(\left\|\mathbf{x}_{c_{i}}-\mathbf{x}_{a}\right\|\right)$

with analogous definitions for $y$ and $z$.

Using RBF interpolation has the advantage of being able to specify the level of control since control points can be placed arbitrarily in, on the boundary, or outside the fluid domain. However, irrespective of the location of the control points, modal deformations defined on the aerofoil surface may not be coincident with the control points so control point deformations must be defined. The authors (Poole et al. 2015b) showed a number of techniques for achieving this, and an inverse RBF interpolation is an effective approach. The set-up of control points around the wing used in this paper is shown in Fig. 2.

Modal deformations are applied sectionally at ten spanwise stations (on the red control points), whilst intermediate control points (shown in blue) are used to permit smooth spanwise deformations between the deformation slices (Allen et al. 2018). The deformation of intermediate points uses a partition of unity-blend of the deformation slices at either side. To define deformations of a slice of intermediate control points, $\Delta_{i}$, the deformations of the slices of main control points on either side of the intermediate slice $\left(\Delta_{m}\right.$ and $\left.\Delta_{m+1}\right)$ are blended as:

$\Delta_{i}=\Delta_{m} \cos ^{2}\left(\frac{\pi \lambda_{i}}{2}\right)+\Delta_{m+1} \sin ^{2}\left(\frac{\pi \lambda_{i}}{2}\right)$,

where $\lambda_{i}=\left(y_{i}-y_{m}\right) /\left(y_{m+1}-y_{m}\right)$. Hence, it can be seen that if the main deformations are equivalent of two neighbouring sections $\left(\Delta_{m}=\Delta_{m+1}\right)$ then any intermediate slice will have the same deformation. Further, it is therefore simple to also define global deformations, where each deformation slice is deformed by the same mode and scaled by the local chord. This means that wing optimization can be performed using only a handful of design variables. The global approach is given in more detail by Allen et al. (2018).

In addition to the sectional deformations, a global twist deformation is introduced. A linear twist is applied from zero at the root to the twist angle at the tip. Hence, the local angle of rotation, $\gamma$, of a main control point slice located at $y_{m}$ is given by $\gamma\left(y_{m}\right)=\gamma y_{m} / s$.

The control point cage is constructed around the local wing coordinates. For example, Fig. 2 shows the cage

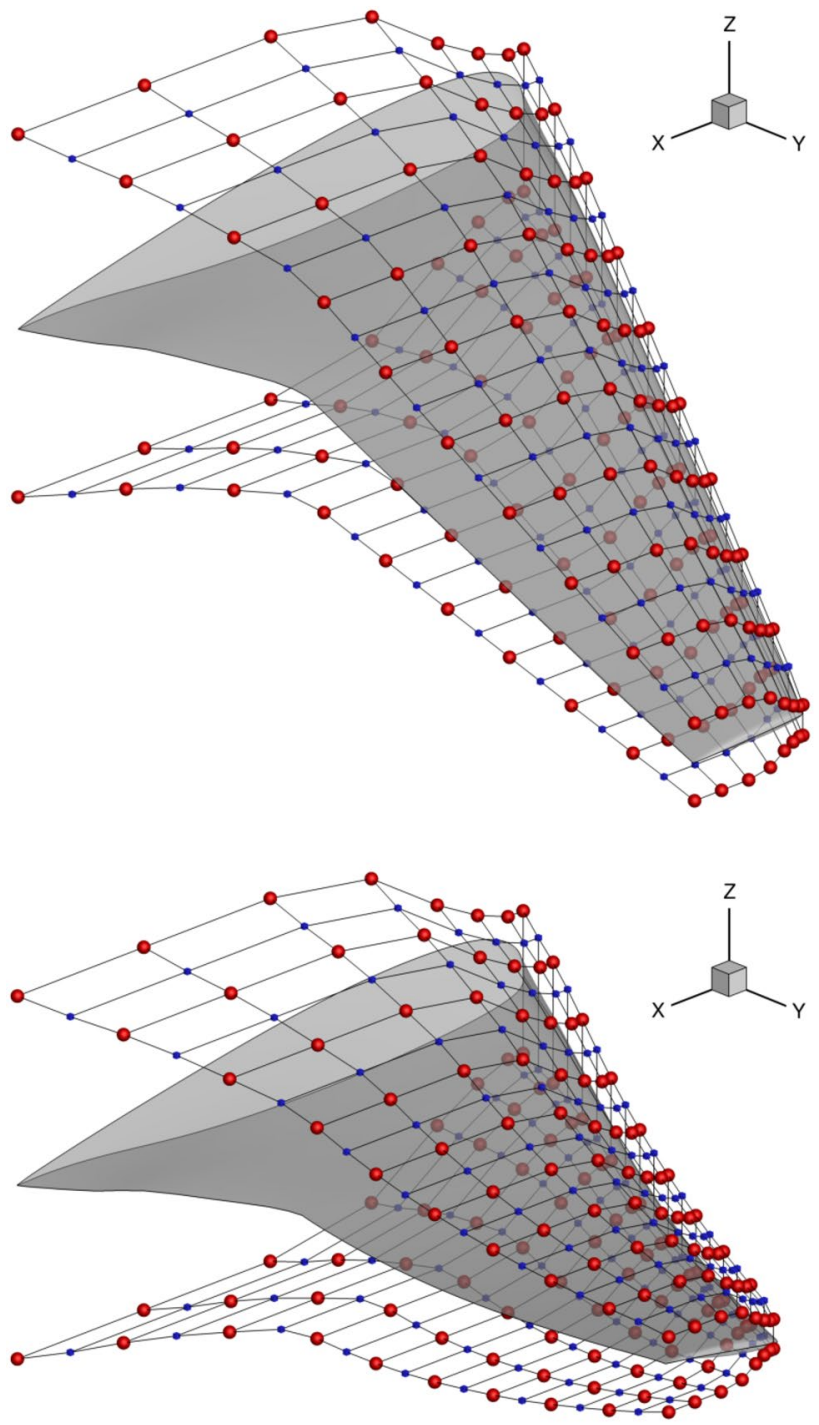

Fig. 2 Control point cages around rigid and aeroelastic wing

around both a rigid wing and a deformed wing. Hence, the sequence of operations is given a design variable vector, $\boldsymbol{\alpha}$, twist deformations of the main sections occurs, followed by sectional deformations at main spanwise stations (if a global deformation is used, then each main spanwise section is deformed by the same mode scaled by the local chord). The intermediate sectional deformations are then calculated. Once all control point deformations are defined and calculated, mesh deformation occurs.

\section{Aero-structural solver and case definition}

The aero-structural solver is based on the structured multiblock solver of Allen $(2002,2006)$, with the coupled solver added through the work of Rendall and Allen (2008). In the 


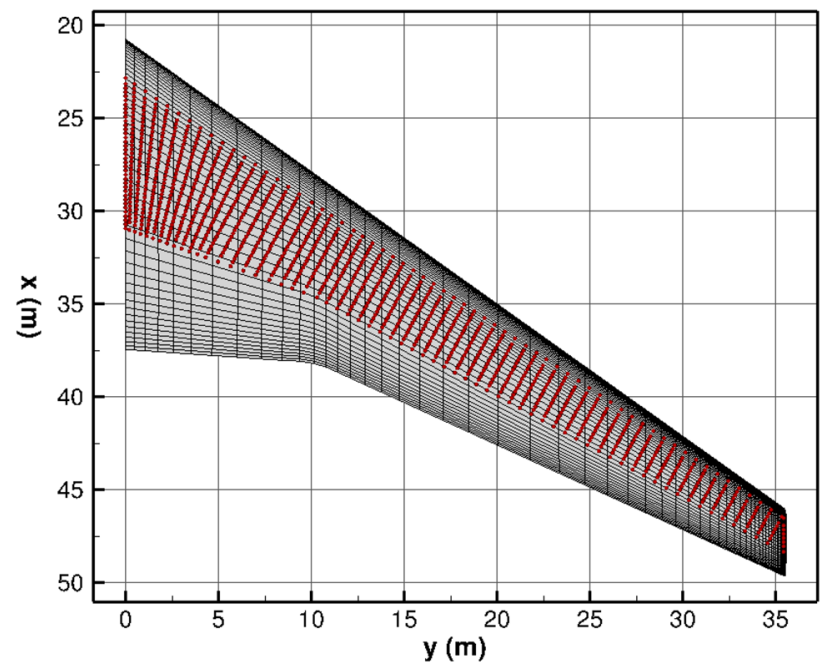

Fig. 3 MDO surface and structural meshes in planform view

case considered in this work, the flow is governed by the Euler equations, which are solved using finite volume integration with the Jameson-Schmidt-Turkel scheme (Jameson et al. 1981). Convergence acceleration is achieved through multigrid (Allen 2001). A modal structural solver is applied where coupling between the aerodynamic and structural grids, and subsequent mesh motion, is achieved through RBF coupling (Rendall and Allen 2008) that uses a reduced point cloud between aerodynamic and structural neighbour nodes (Rendall and Allen 2010). Strong coupling of the two systems is employed for dynamic calculations. Newmark temporal integration (Newmark 1959) is used to march the solution.

To demonstrate using the compact aerofoil decomposition for aero-structural optimization, the MDO wing (Allwright 1997; Haase et al. 2002) is used, where the structural model and associated structural mode shapes are defined. It should be noted that the geometry referred to through this paper as rigid, is defined in Sec 8, Chapter 4 of (Haase et al. 2002) as 'jig-shape'. This had been back calculated from the designed flight shape for the purposes of the aeroelastic computations of the UNSI project. The aeroeastic solutions in this paper represent the calculated flight shape at the specified loading condition. Figure 3 shows the MDO wing planform. Also shown is the aerodynamic surface mesh and the structural grid. A 2515 node wing-box structural model is used with the modes defined by Haase et al. (2002).

Throughout this work, a trimmed cruise condition of $C_{L}=0.4$ at $M_{\infty}=0.85$ is used. Unless otherwise stated, all wings are trimmed to this condition. The MDO wing has 18 defined structural modes and all are used for this work.

To ensure sufficient aerodynamic resolution, a mesh dependence study is presented. A family of eight-block

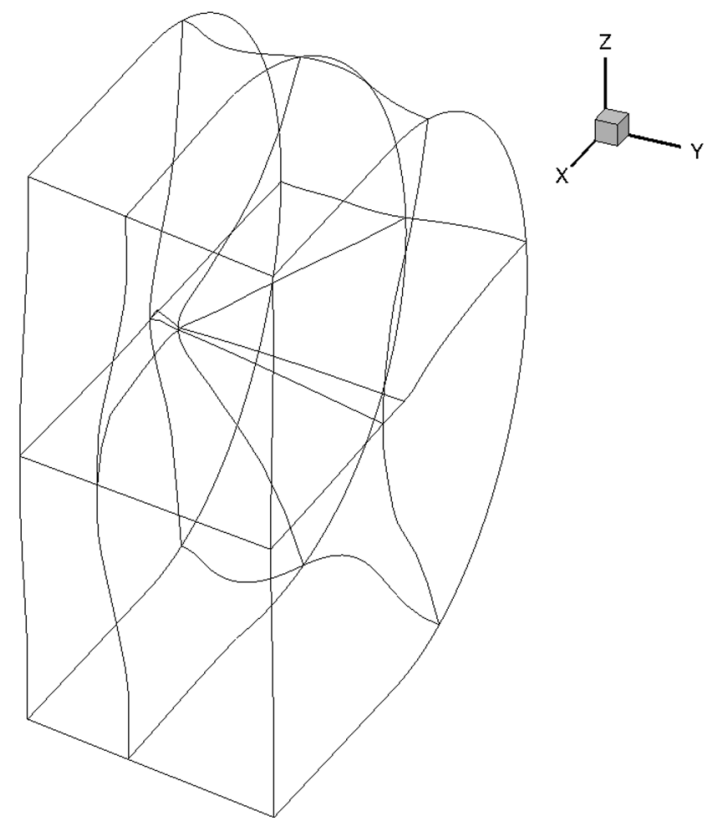

Fig. 4 Block structure of eight-block structured C-meshes

structured C-meshes (block structure is given in Fig. 4 was generated using the methods of Allen (2008) to give high quality meshes. These range in size from 2.1 million to 0.13 million cells, and are designated L1 (2.1M), L2 (1.1M), L3 (580k), L4 (260k) and L5 (130k); sizes were chosen with approximately a two-times scaling between mesh levels, and to maximise the number of multigrid levels for each mesh.

Each mesh was used to produce both a rigid and aeroelastic solution and the final force coefficients of each run are given in Table 1 . The structural deflection is calculated based on the deformation of the structural node closest to the aerodynamic tip. Also given are the run-times relative to the run-time of the coarsest mesh, with the runs being performed in serial to obtain comparable figures. All wings were run at a trimmed cruise $C_{L}$ of 0.4 . Figure 5 gives the surface pressure coefficients of the coarsest and finest meshes. Clearly, there is little difference in the flowfields, and the structural deformations are almost identical. The L4 mesh, whilst being relatively coarse, appears to be a good compromise between run-time and accuracy whilst providing accurate capture of the structural deformation field.

The primary mesh used for the study is the L4 mesh, shown in Fig. 6, which contains 273k nodes. This mesh has a $97 \times 57$ surface mesh, 21 nodes on either side of the wake, and 25 nodes between the inner and outer boundary.

Figure 7 shows the solution of the rigid MDO wing and the aeroelastic wing (i.e. in flight shape). and Fig. 8 gives the span-wise loading distribution. There is a strong stock present on the rigid wing, that when in flight shape is no 
Table 1 Force coefficients and run-times (relative to L5 mesh) on different meshes

\begin{tabular}{|c|c|c|c|c|c|c|c|}
\hline \multirow[t]{2}{*}{ Mesh } & \multicolumn{3}{|c|}{ Rigid } & \multicolumn{4}{|c|}{ Aeroelastic } \\
\hline & $C_{\mathrm{L}}$ & $C_{\mathrm{D}}$ & $\begin{array}{l}\text { Run-time } \\
\text { (×L5) }\end{array}$ & $\overline{C_{\mathrm{L}}}$ & $C_{\mathrm{D}}$ & $\Delta z_{\text {struc }}$ & $\begin{array}{l}\text { Run-time } \\
(\times \text { L5) }\end{array}$ \\
\hline L5 & 0.4 & 0.0192 & 1.0 & 0.4 & 0.0211 & $6.15 \mathrm{~m}$ & 1.0 \\
\hline L4 & 0.4 & 0.0158 & 2.2 & 0.4 & 0.0177 & $6.18 \mathrm{~m}$ & 1.7 \\
\hline L3 & 0.4 & 0.0134 & 4.9 & 0.4 & 0.0153 & $6.19 \mathrm{~m}$ & 3.9 \\
\hline L2 & 0.4 & 0.0121 & 7.2 & 0.4 & 0.0137 & $6.10 \mathrm{~m}$ & 7.4 \\
\hline L1 & 0.4 & 0.0119 & 11.9 & 0.4 & 0.0134 & $6.12 \mathrm{~m}$ & 11.8 \\
\hline
\end{tabular}

Fig. 5 Surface $C_{\mathrm{P}}$ of aeroelastic wing shape on L5 and L1 meshes
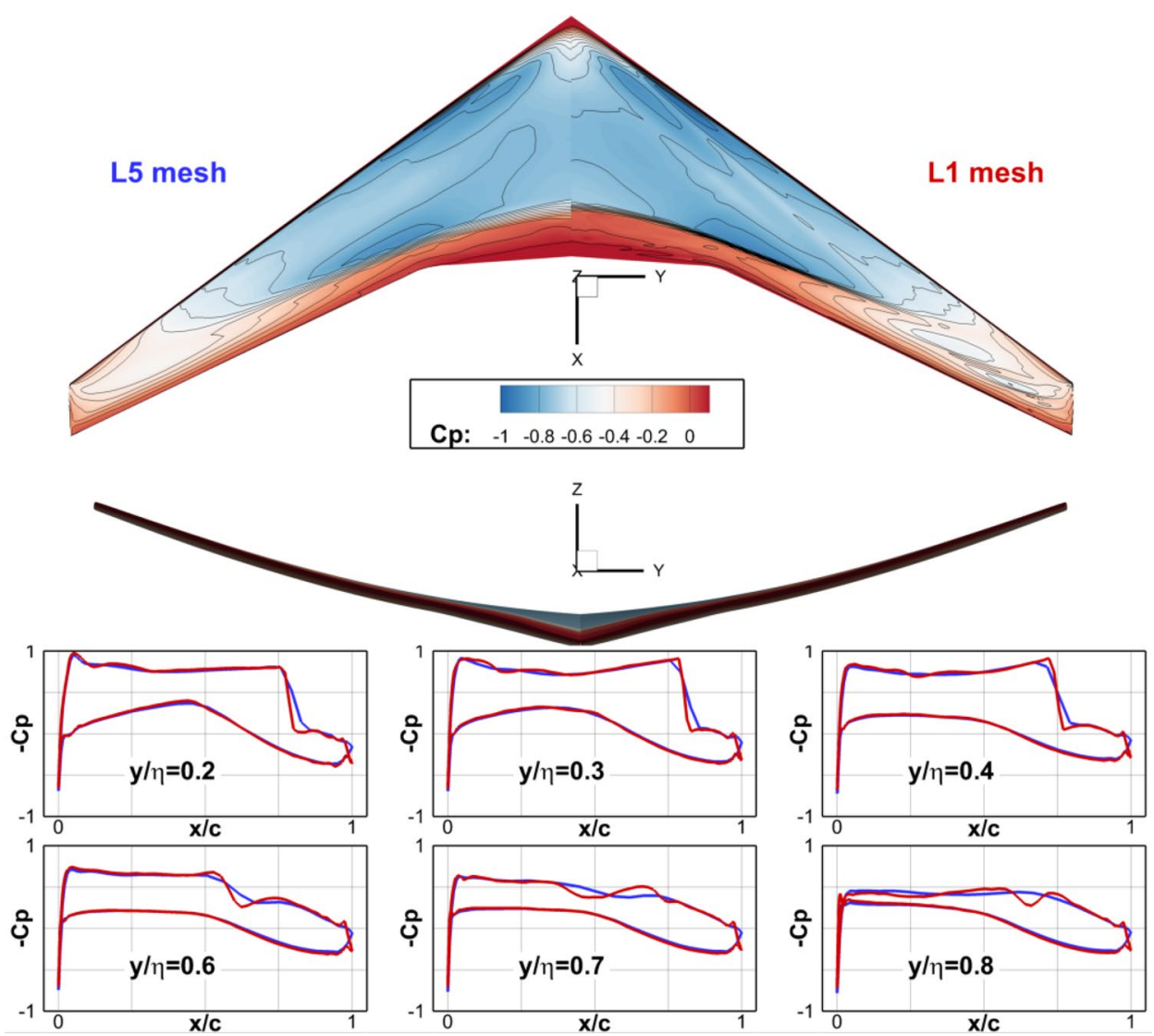

longer present outboard. The loading is shifted inboard and the distribution is almost perfectly triangular in flight shape. This results in a lower root bending moment at the expense of a drag penalty.

\section{Optimization problem and algorithm}

A generic single-objective optimization problem optimizes a cost function, $J$, which is a function of a vector of $D$ design variables, $\boldsymbol{\alpha}$, subject to a vector of inequality constraints, $\mathbf{g}$, and equality, $\mathbf{h}$, constraints. The problem considered in this paper is aerodynamic shape optimization. The objective is drag minimization subject to constraints on lift coefficient, $C_{L}$ and internal volume $V$. The problem is given by:

$$
\begin{aligned}
& \underset{\alpha \in \Re^{D}}{\operatorname{minimise}} \quad C_{D} \\
& \text { subject to } \\
& V \geq V \text { (initial) }
\end{aligned}
$$

The design variables of the problem are aerofoil deformation modes applied at ten equally spaced sections across the span of the wing. Furthermore, to allow induced drag to reduce, 

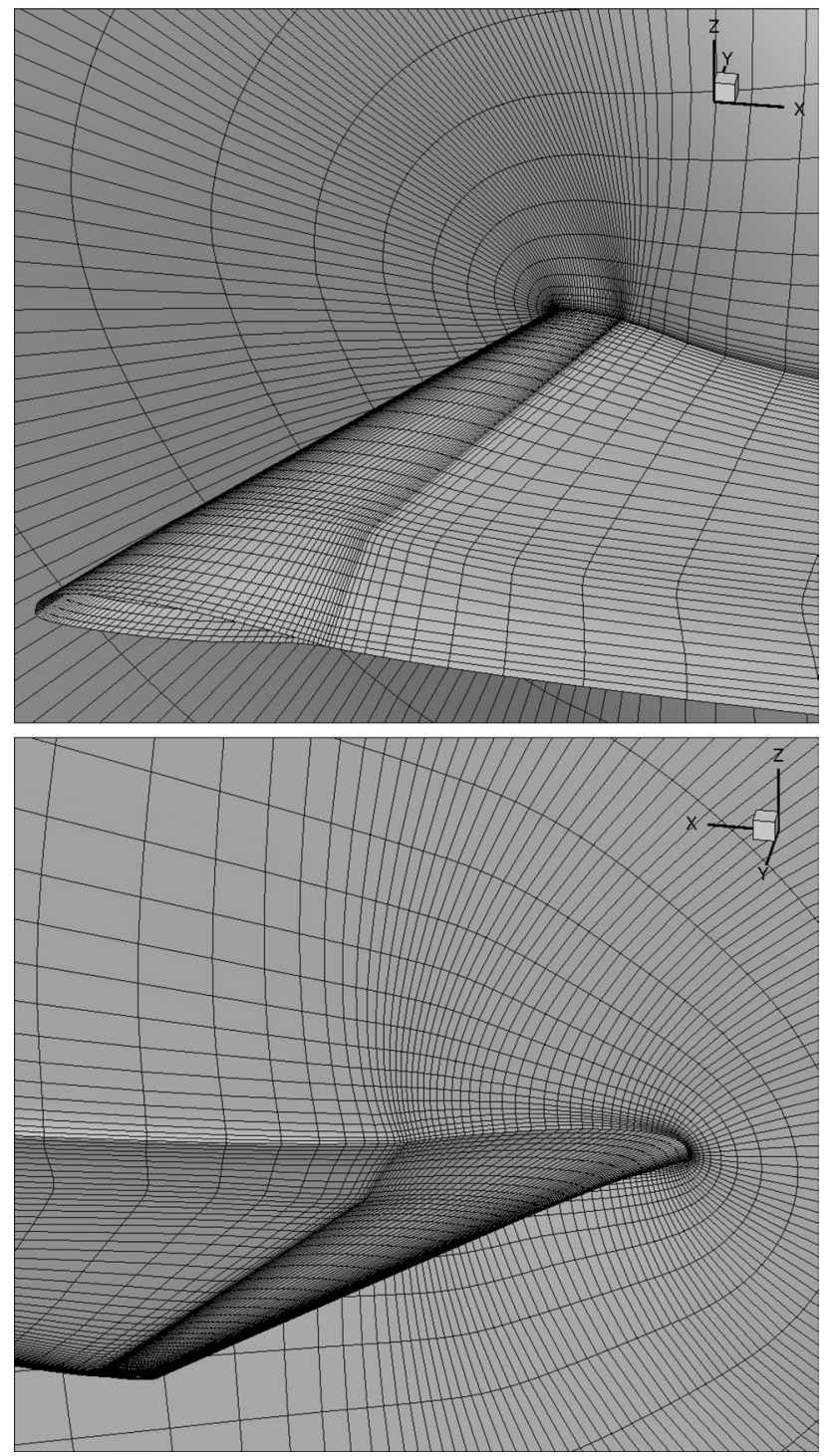

Fig. 6 L4 mesh

the linear (root-to-tip) twist variable is used, and to allow balance of the lift loading, angle of attack is also a design variable. For the aeroelastic optimizations, both local and global modal deformations are considered. Details of the different design variables are given in Table 2.

Using structural modal analysis is an effective means to determine the coupled aero-structural performance of the wing. However, when used in an optimization process, the primary assumption is that modifying the aerodynamic shape has minimal effect on the underlying structural model so the same structural modes can be used. This assumption holds assuming the structure is not changed, and since a wing-box is modelled, no planform changes occur but only minor surface changes, this is reasonable.

In the case considered in this paper, the overall goal of this work is to permit large-scale global aeroelastic shape optimization. Global optimization is particularly useful when the design space is known to be multimodal, and the overall goal is to locate the globally optimal solution. In the first instance, only sectional changes are considered and since fixed planform wing optimization is generally considered to be unimodal (to within numerical tolerances) ( $\mathrm{Yu}$ et al. 2018), the gradient-based optimization algorithm, feasible sequential quadratic programming (FSQP) algorithm as implemented in version 3.7 (Zhou et al. 1997), is used. FSQP is based on the sequential quadratic programming (SQP) approach, but modified to avoid the so-called 'Maratos' effect (restriction of a step size due to the requirement of feasibility) (Maratos 1978), by combining a search along an arc (Mayne and Polack 1982) with a non-monotone procedure for that search (Grippo et al. 1986). The non-monotone line search relaxes the rule that a step must result in an improvement in the objective function at each major iteration, and instead requires an improvement over the best of the last four iterations. This means that the objective function can occasionally worsen from one iteration to the next. The Hessian is updated via the Broyden-Fletcher-GoldfarbShanno (BFGS) scheme, with identity initialisation. As is common with gradient-based optimization, the convergence is not based on a fixed number of iterations, rather on ensuring either convergence of the Karush-Kuhn-Tucker (KKT) conditions, or no change in the objective function within the non-monotone procedure, whichever occurs first. The FSQP algorithm is fully described and analysed in (Panier and Tits 1991; Bonnans et al. 1992).

The gradient-based optimizer requires the sensitivities of the cost and constraint functions with respect to each design variable at each major iteration. For this work, a secondorder finite difference stencil is used so for each design variable, two extra flow solutions are required (one each for the positive and negative perturbations) to evaluate the sensitivities. For computational efficiency, a parallel decomposition of the gradient evaluation is employed such that each design variable sensitivity is assigned to its own CPU, which 
Fig. 7 Surface $C_{\mathrm{P}}$ of rigid and aeroelastic wing shapes on optimization mesh (L4)
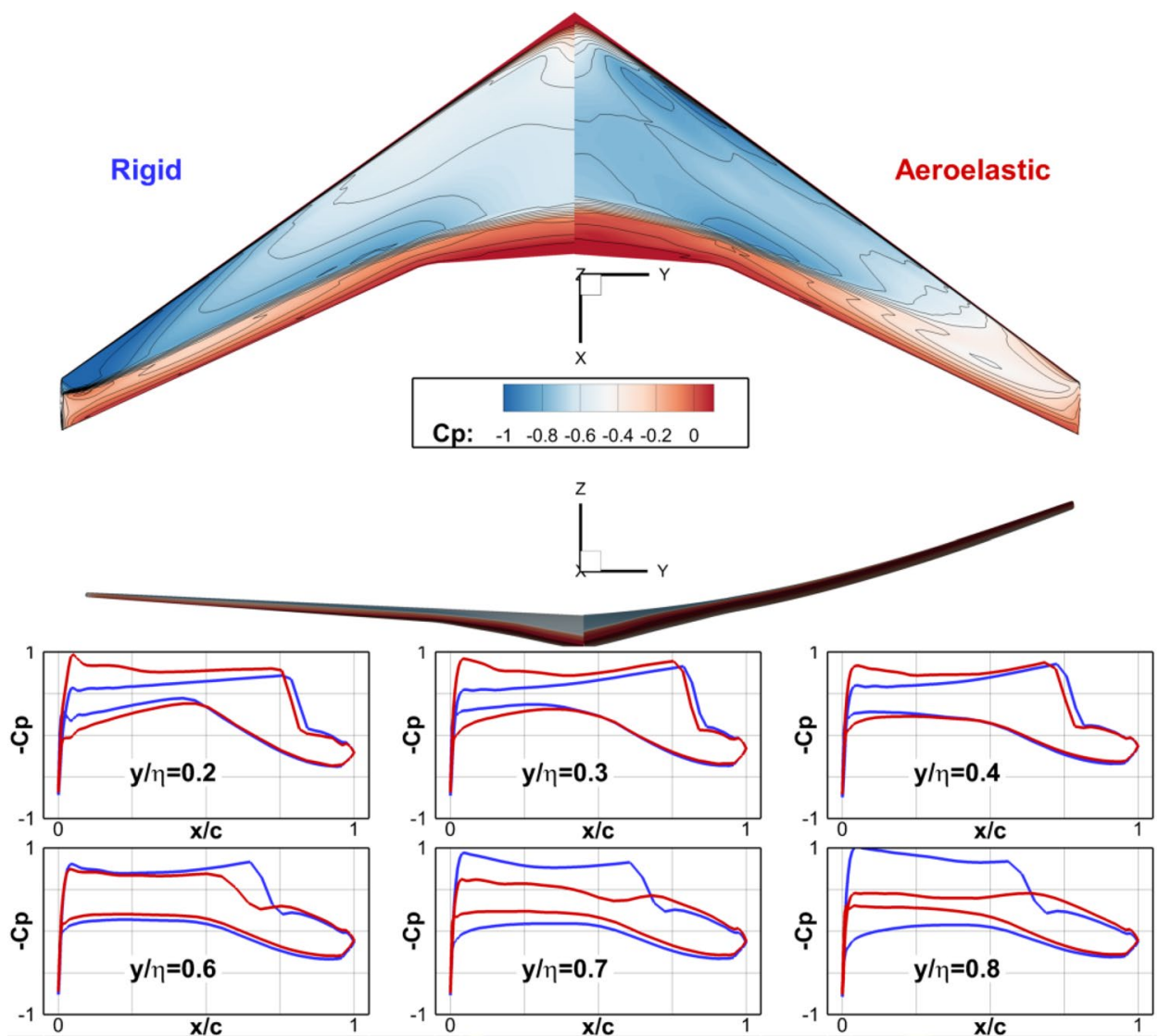

Table 2 Numbers of design variables

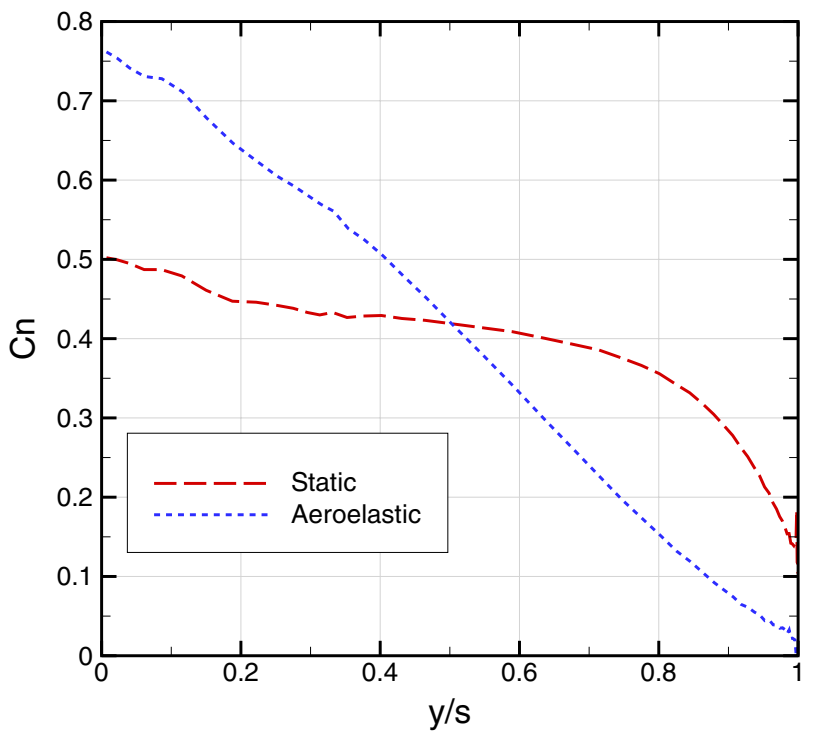

\begin{tabular}{llc}
\hline Global & Local & Total \\
\hline 2 (twist, angle) & $\begin{array}{l}40(4 \text { modes/section } \times 10 \text { sec- } \\
\text { tions })\end{array}$ & 42 \\
2 (twist, angle) & $\begin{array}{l}60(6 \text { modes/section } \times 10 \mathrm{sec}- \\
\text { tions })\end{array}$ & 62 \\
2 (twist, angle) & $80(8$ modes/section $\times 10 \mathrm{sec}-$ & 82 \\
& $\quad$ tions $)$ & 6 \\
2 (twist, angle $)+4$ modes & 0 & 8 \\
2 (twist, angle $)+6$ modes & 0 & 10 \\
2 (twist, angle $)+8$ modes & 0 & \\
\hline
\end{tabular}

handles the geometry (and CFD volume mesh) deformations and flow solutions. Once the gradients are evaluated, these are passed back to the master process where the optimizer update occurs.

Fig. 8 Span-wise loading on MDO wing 
Table 3 Optimization results of rigid MDO wing

\begin{tabular}{lllllll}
\hline & $C_{\mathrm{L}}$ & $C_{\mathrm{D}}$ & $C_{\mathrm{M}_{x}}$ & $C_{\mathrm{M}_{y}}$ & $V\left(\mathrm{~m}^{3}\right)$ & $\Delta C_{\mathrm{D}}$ \\
\hline Initial & 0.4 & 0.0158 & 0.183 & -0.476 & 241.0 & - \\
42 variables & 0.4 & 0.0127 & 0.172 & -0.451 & 241.0 & $\mathbf{- 1 9 . 6 \%}$ \\
62 variables & 0.4 & 0.0125 & 0.173 & -0.457 & 241.0 & $\mathbf{- 2 0 . 8 \%}$ \\
82 variables & 0.4 & 0.0124 & 0.173 & -0.457 & 241.0 & $\mathbf{- 2 1 . 5 \%}$ \\
\hline
\end{tabular}

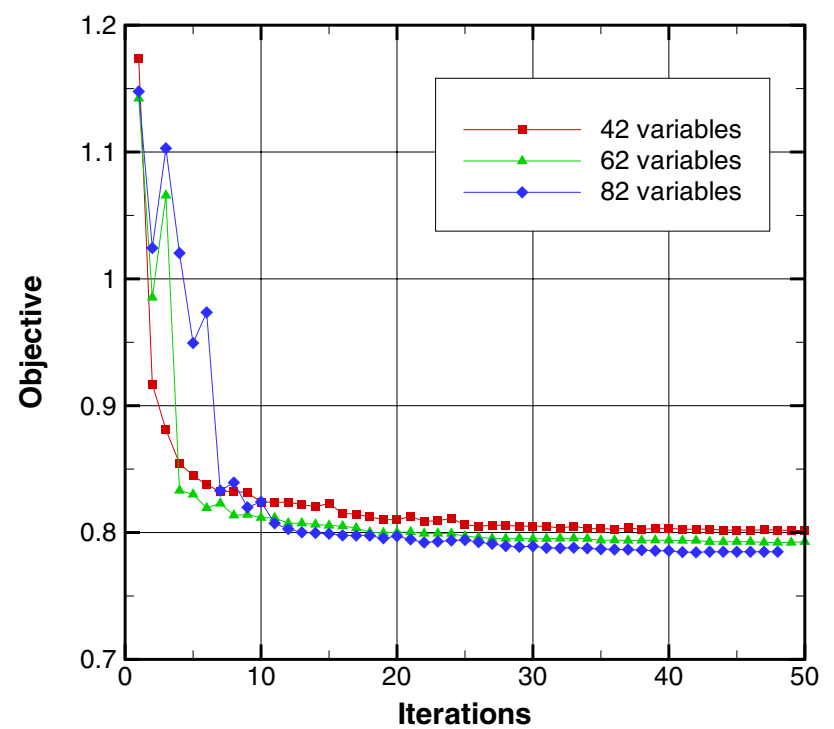

Fig. 9 Optimizer convergence for rigid MDO wing

\section{Rigid wing optimization results}

Initially, to determine both the optimization set-up and a suitable number of SVD modal design variables, optimization of the rigid MDO wing is performed at the trimmed cruise flight condition $\left(C_{L}=0.4\right.$ at $\left.M_{\infty}=0.85\right)$. This is performed using the local modal deformation cases only (i.e. 42, 62 and 82 variables), with global modes (i.e. 6, 8 and 10 variables) applied to the aeroelastic case to demonstrate the flexibility of this approach.

Table 3 gives the optimization results of the optimizations, whilst Fig. 9 gives the convergence of the optimizer. For all three of the design variable combinations tested, both constraints are active and the drag has reduced substantially. Of particular interest is the monotonic decrease in the acquired objective function with increasing number of design variables. In the geometric space, the modal design variables are perfectly orthogonal, which has the implication that the design space of lower numbers of design variables is contained within the design space of higher numbers. In the aerodynamic space, this is not necessarily the case; though the authors have shown this to be the case for aerofoil optimization (Poole et al. 2017). Albeit, the monotonic decrease indicates that geometrically orthogonal aerofoil design variables exhibit similar performance when applied to wing optimization.

Figure 10 shows the surface pressures of the baseline wing and 82 variables optimized wing, and demonstrates that the result is shock-free at the design condition. Since this problem is inviscid, the two primary sources of drag are wave drag and induced drag. As the problem is shock-free, wave drag has been substantially reduced.

\subsection{Aeroelastic solution of optimised rigid wing}

The rigid optimizations have produced the expected shockfree result at the design condition, however, it is interesting to see how this wing performs under aeroelastic loading. Hence, the optimized wing is run with the structural model to determine the aeroelastic solution. Table 4 gives the force coefficients of the aeroelastic MDO wing and the aeroelastic solution of the optimized rigid wing, whilst Fig. 11 shows the surface pressure coefficients. It should be noted that all wings are trimmed to $C_{\mathrm{L}}=0.4$. Clearly the aeroelastic solution of the optimized rigid wing has a shock structure, with a single shock at the inboard and outboard sections, and a double shock midspan and this has resulted in an overall drag increase. Also, the extra outboard load of the rigid optimized wing has resulted in a lower tip deflection.

It is well known that single-point drag minimization produces highly optimized point-design solutions with poor off-design performance (Poole et al. 2018a), so an increase in drag for the aeroelastic solution of a rigid wing is not surprising. The changes in loading that occur due to shape changes in optimization, lead to a change in the deflected 
Fig. 10 Surface $C_{\mathrm{P}}$ of initial and optimized wing shapes for 82 variables case
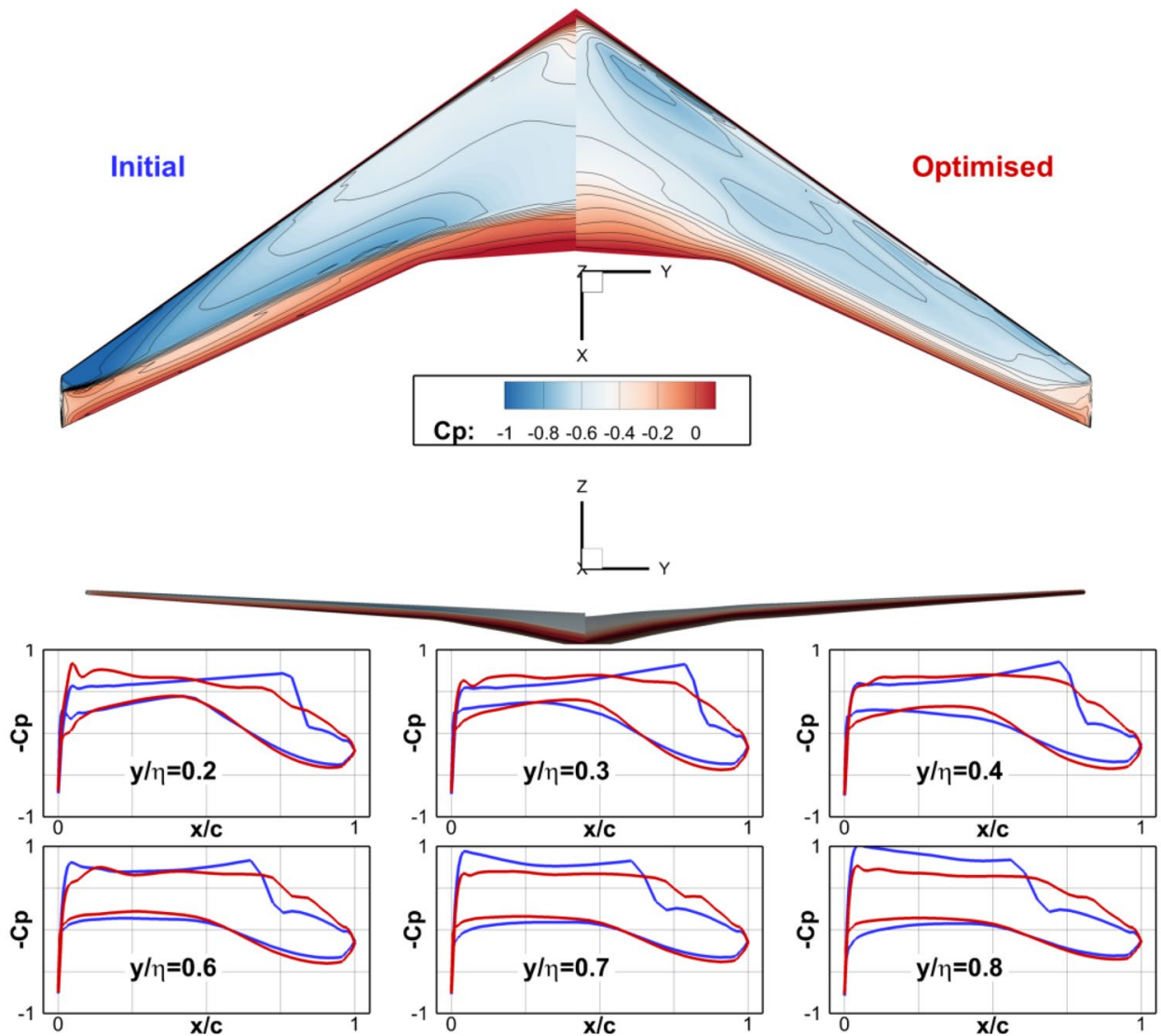

\subsection{Local modal deformations}

Table 4 Forces of aeroelastic MDO wing and aeroelastic solution of optimized rigid wing

\begin{tabular}{llllll}
\hline & $C_{\mathrm{L}}$ & $C_{\mathrm{D}}$ & $C_{\mathrm{M}_{x}}$ & $C_{\mathrm{M}_{y}}$ & $\Delta z_{\text {struc }}(\mathrm{m})$ \\
\hline MDO & 0.4 & 0.0177 & 0.143 & -0.382 & 6.18 \\
Optimized & 0.4 & 0.0203 & 0.138 & -0.375 & 5.33 \\
\hline
\end{tabular}

shape which leads to a further change in the loading. It is therefore not enough to consider optimizing a wing statically, but that the full aero-structural solution must be taken into account to obtain any meaningful results.

\section{Aeroelastic wing optimization results}

As a result of the experiment presented above, it is clear that wing optimizations should contain wing shape responses also. In that light, aerodynamic shape optimizations are presented of the aeroelastic MDO wing.
Table 5 shows the optimization results for varying numbers of locally applied modes, and Fig. 12 gives the optimizer convergence. As in the rigid case, monotonic decrease in the objective function is observed, which is to be expected with the modal design variables, but is encouraging to observe. Both the lift and volume constraints are active for all the cases. The tip deflection has increased substantially for the optimized wing indicating that transferring load from root to tip (the load is almost perfectly triangular in the MDO wing, but close to elliptical in the optimized wing) and allowing a more flexible solution (something observed in modern transonic aircraft) permits shock strength reduction. This is demonstrated in the surface pressure shown in Fig. 13.

Comparing the final optimized shapes for both the rigid and aeroelastic optimizations, Fig. 14 shows the surface changes made by the optimizer to the wing shape for both the upper and lower surfaces. It is clear that there are substantial differences in the final optimized shapes. The rigid wing case has pushed the trailing edge up, whereas the 
Fig. 11 Surface $C_{\mathrm{P}}$ of MDO wing and rigid optimization result under aeroelastic loading
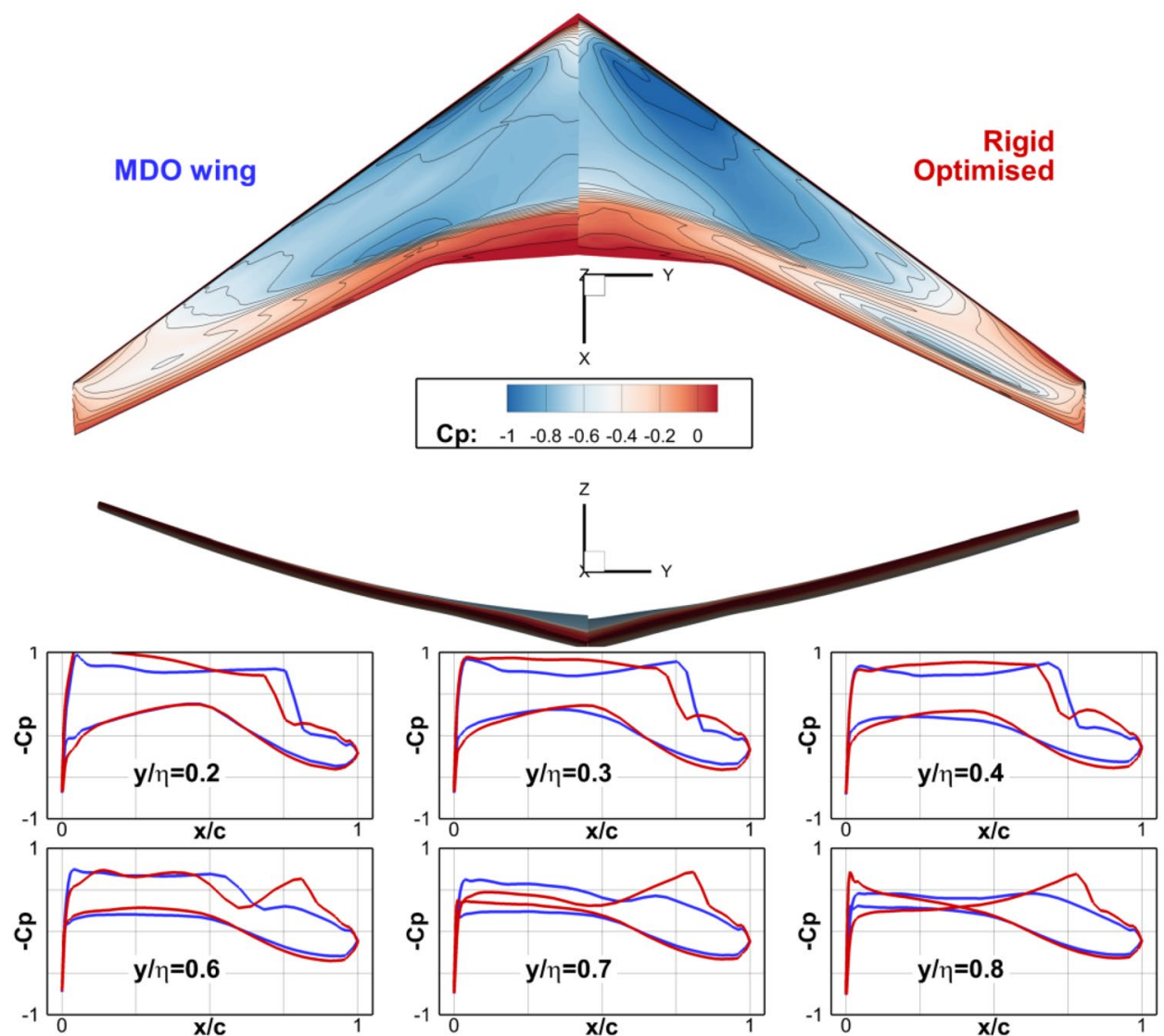

Table 5 Optimization results of aeroelastic MDO wing (local sectional deformations)

\begin{tabular}{lllllllc}
\hline & $C_{\mathrm{L}}$ & $C_{\mathrm{D}}$ & $C_{\mathrm{M}_{x}}$ & $C_{\mathrm{M}_{y}}$ & $V\left(\mathrm{~m}^{3}\right)$ & $\Delta z_{\text {struc }}(\mathrm{m})$ & $\Delta C_{D}$ \\
\hline Initial & 0.4 & 0.0177 & 0.143 & -0.382 & 241.0 & 6.18 & - \\
42 variables & 0.4 & 0.0126 & 0.184 & -0.436 & 241.0 & 9.39 & $\mathbf{- 2 8 . 8 \%}$ \\
62 variables & 0.4 & 0.0122 & 0.187 & -0.447 & 241.0 & 9.78 & $\mathbf{- 3 1 . 1 \%}$ \\
82 variables & 0.4 & 0.0121 & 0.187 & -0.450 & 241.0 & 9.71 & $\mathbf{- 3 1 . 6 \%}$ \\
\hline
\end{tabular}

aeroelastic case has pushed this down. Furthermore, the magnitude of these deformations are much larger in the aeroelastic case than the rigid one. It is therefore not surprising that a rigid optimization subsequently run elastic, produces a completely different solution to an optimization that is run using the elastic case. It is therefore clear that to perform wing optimizations, the coupling between aerodynamic forces and structural response must be included.

\subsection{Global modal deformations}

Finally, a study is presented on an approach for performed extremely efficient aeroelastic wing optimization. The use of the framework developed in this paper means that aerofoil modal deformations can be applied across the whole wing (scaled by the local chord). This results in extremely low-dimensional wing optimization problems, with an order 


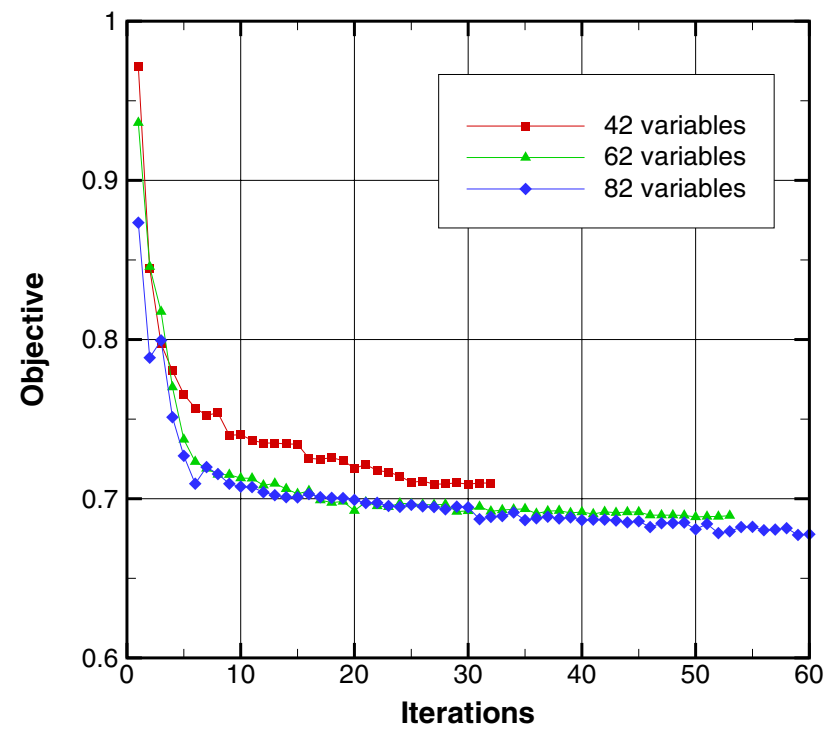

Fig. 12 Optimizer convergence for aeroelastic MDO wing (local sectional deformations) of magnitude fewer design variables than including local deformations. Such lower number of variables does mean that very localised deformations of the surface are not permissible, but it is interesting to consider whether performing fixed planform, sectional optimization of elastic wings is permissible with only a few design variables.

Table 6 gives the final optimization results for the global sectional deformation runs, whilst the optimizer convergence is given in Fig. 15. Like the local runs, a monotonic decrease in the objective function (drag) is observed, which is expected since use of these modal parameters leads to the design spaces of lower numbers of modes being entirely enclosed within that of higher numbers of modes. Furthermore, it is extremely encouraging to see that large drag reductions are still possible with a very small number of design variables (especially for full 3-D wing optimization). The optimizer converges rapidly, though like in the local cases, a small 'tail' of the convergence is seen. This can be common in aerodynamic optimization, where the optimizer is tweaking the final surface to reduce the small amount of
Fig. 13 Surface $C_{\mathrm{P}}$ of initial and optimized wing shapes for 82 variable case
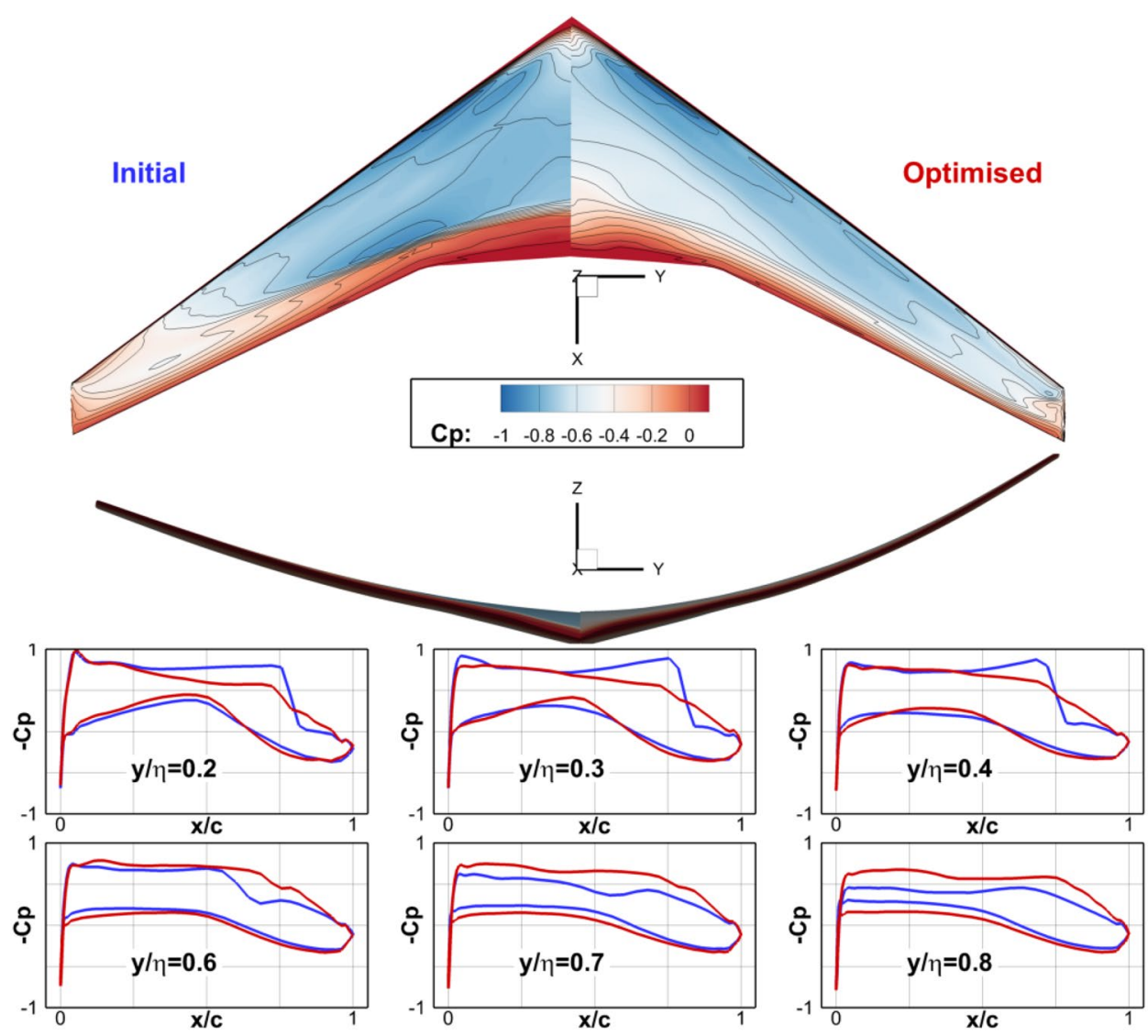
Fig. 14 Signed-magnitude contours of the surface deformation (positive is away of the surface) showing difference between optimized wings and the baseline (before aeroelastic deformation)

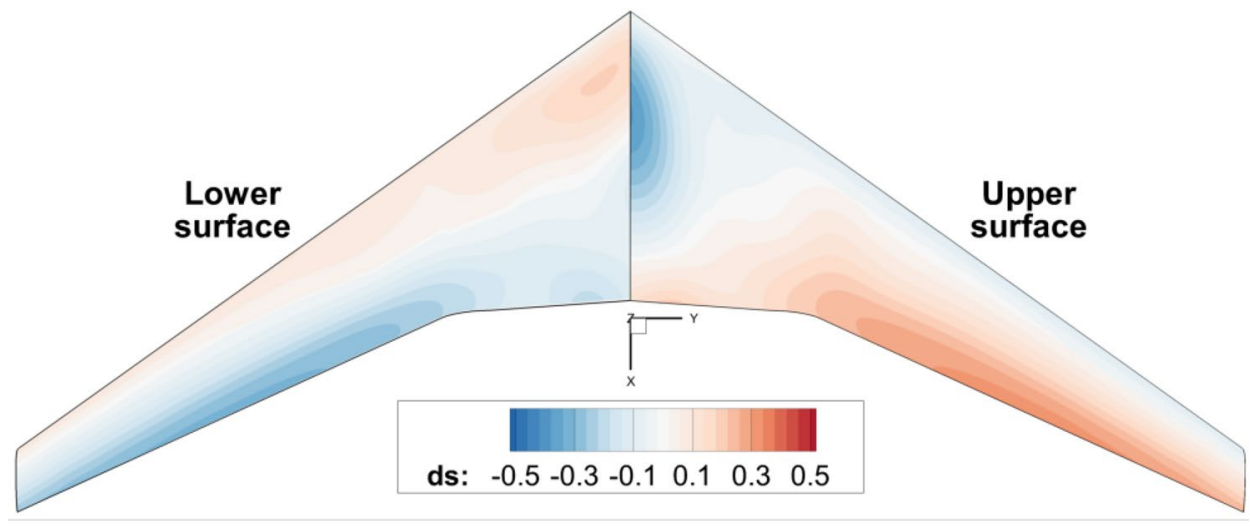

(a) 82 variables rigid optimization

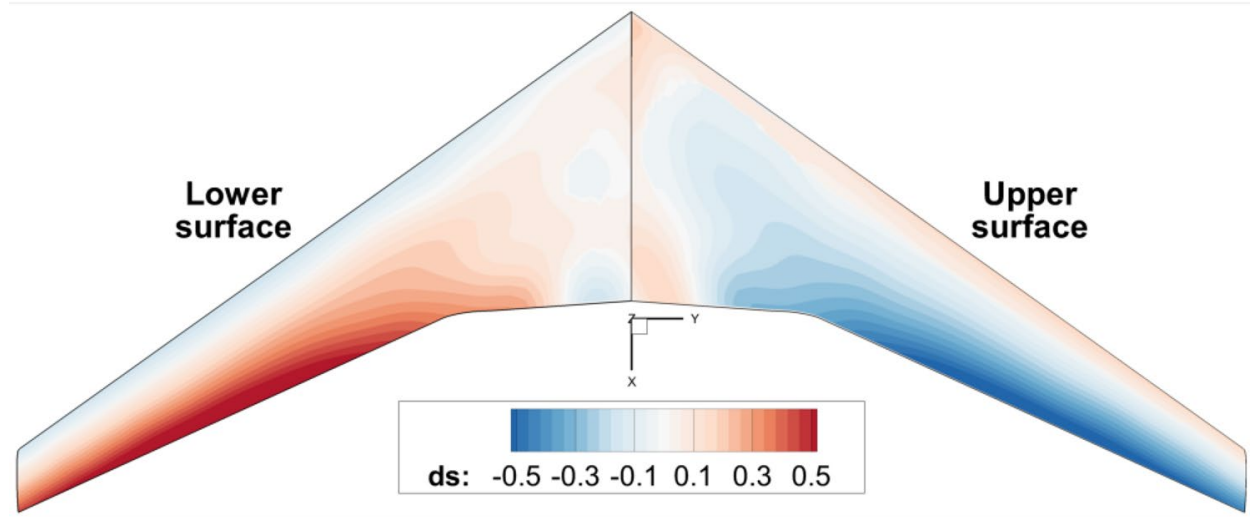

(b) 82 variables aeroelastic optimization

\begin{tabular}{llllllll}
\hline & $C_{\mathrm{L}}$ & $C_{\mathrm{D}}$ & $C_{\mathrm{M}_{x}}$ & $C_{\mathrm{M}_{y}}$ & $V\left(\mathrm{~m}^{3}\right)$ & $\Delta z_{\text {struc }}(\mathrm{m})$ & $\Delta C_{\mathrm{D}}$ \\
\hline Initial & 0.4 & 0.0177 & 0.143 & -0.382 & 241.0 & 6.18 & - \\
6 variables & 0.4 & 0.0132 & 0.180 & -0.442 & 241.0 & 9.22 & $\mathbf{- 2 5 . 4 \%}$ \\
8 variables & 0.4 & 0.0129 & 0.177 & -0.447 & 241.3 & 9.06 & $\mathbf{- 2 7 . 1 \%}$ \\
10 variables & 0.4 & 0.0128 & 0.176 & -0.444 & 241.1 & 8.97 & $\mathbf{- 2 7 . 7 \%}$ \\
\hline
\end{tabular}

Table 6 Optimization results of aeroelastic MDO wing (global sectional deformations) reduction in the number of iterations the optimiser required to converge. This is particularly prevalent in the 8 modes cases, where global application of the modes shows a $70 \%$ reduction in the cost of the optimization, with only a small drag penalty. 


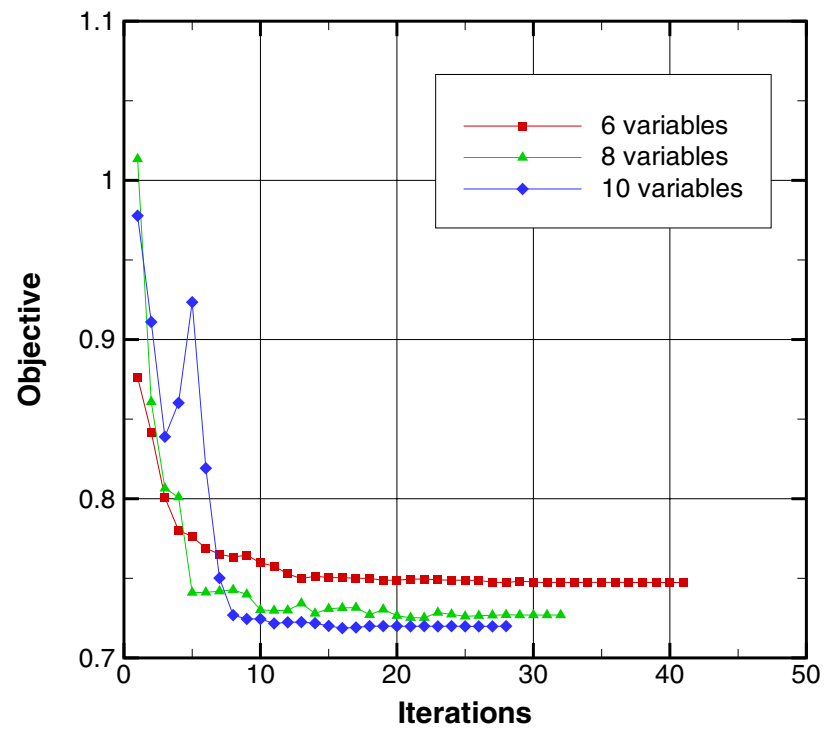

Fig. 15 Optimizer convergence for aeroelastic MDO wing (global sectional deformations)

Table 7 Total CFD solver calls for aeroelastic optimizations

\begin{tabular}{lrr}
\hline SVD modes & \multicolumn{2}{l}{ CFD runs } \\
\cline { 2 - 3 } & \multicolumn{1}{c}{ Local } & Global \\
\hline 4 & 6630 & 3185 \\
6 & 13250 & 3366 \\
8 & 23925 & 6993 \\
\hline
\end{tabular}

Figure 16 compares the surface pressures for the 8 aerofoil modes applied locally ( 82 variable case) and globally (10 variable case). Broadly, the pressure distributions are very similar, which is encouraging. Figure 17 compares the loading of these wings showing similar shapes, though with the local modes having the fidelity to shift loading outboard (and therefore also have a slightly higher tip deflection). Due to the fidelity of the local deformations, these have successfully managed to eliminate the shock, whilst in the global case, this is not the case. However, comparing the final drag values, the difference between the two cases is only seven drag counts, which is $4 \%$ of the initial drag. This is a performance penalty over the local deformations, though it should be noted that this is inviscid flow so if viscous drag was included, the percentage penalty would be different. Still, it shows that a highly compact design space is still explorable with these modes. The low number of modes has not had an appreciably negative effect on the aeroelastic solution demonstrating that extremely low-dimensional flexible wing optimization is a reality. Having such a low number of design variables for elastic wing optimization opens up the possibility of introducing global optimization methods (where a very low dimensional design space is necessary) to high-fidelity wing optimization, particularly where coupling may occur between sectional and planform changes.

A final comparison of all the wings considered here is shown in Fig. 18, emphasising the different in flight shapes of the optimised wings.

\section{Conclusions}

Efficient multi-disciplinary analysis and subsequent optimization have been presented for aeroelastic wing optimization using an efficient low-dimensional surface deformation scheme. Whilst much aero-structural optimization work to date has considered high-fidelity, high-dimensional problems, little work has considered the possibility of reducing the dimensionality of the problem. As such, the authors have previously presented an efficient method for determining aerofoil deformations via singular value decomposition. The resulting design variables are geometrically orthogonal resulting in a well-conditioned design space. The work presented in this paper has developed an approach to apply these to elastic wings in such a way that the aerofoil deformations can be applied locally to each section or globally (scaled by the local chord). This flexibility allows the user to perform localised optimization using many design variables, but also low-dimensional optimization through global sectional deformations.

The compact aerofoil design variables were applied sectionally for inviscid drag minimization subject to lift and internal volume constraints. The MDO wing was considered, with optimizations presented on the rigid wing and elastic wing. A gradient-based optimization approach with parallel gradient evaluation was utilised. Shock-free solutions were demonstrated for the rigid wing, indicating suitability of the aerofoil modes for sectional-based wing optimization. However, it was demonstrated that a wing designed in rigid shape had poor performance when loaded in flight shape. Hence, the optimization framework was applied to the aeroelastic wing to produce shock-free solutions. The resulting shapes had a much increased tip deflection. The orthogonality 
Fig. 16 Comparison of surface $C_{\mathrm{P}}$ of optimized wing shapes for eight aerofoil modes applied locally ( 82 variables case) and globally (10 variable case)

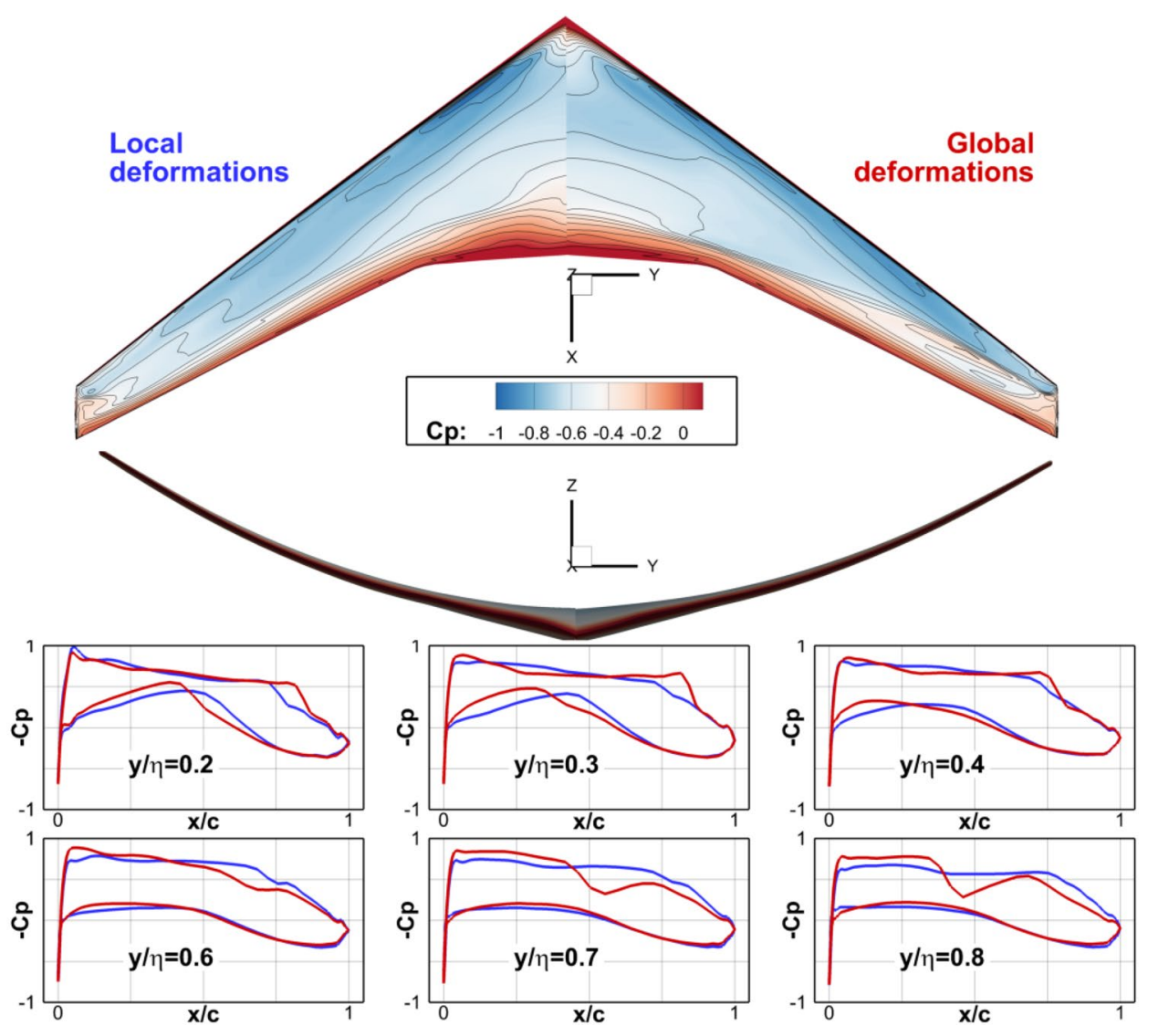

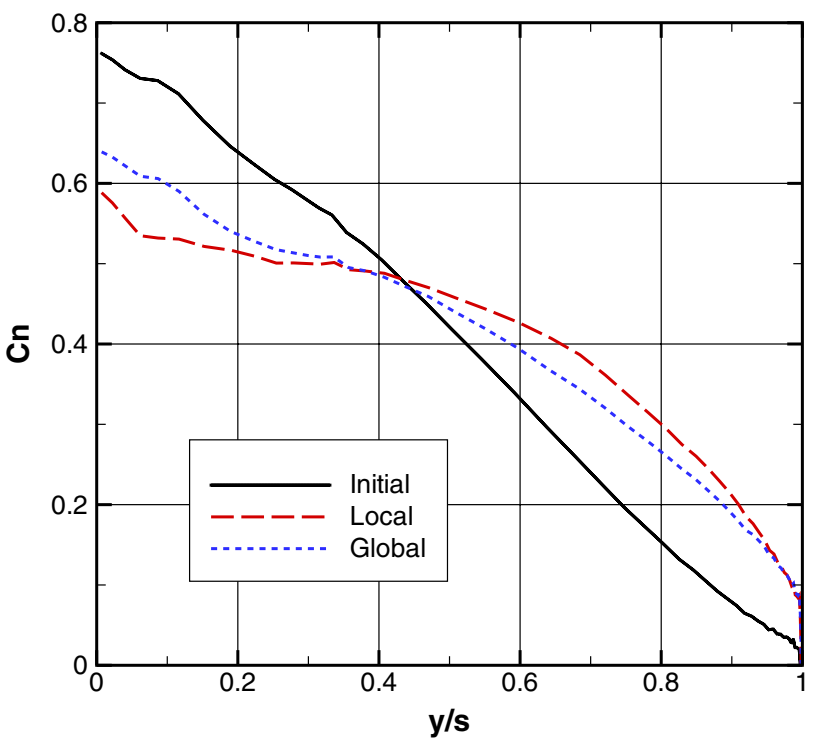

Fig. 17 Comparison of span-wise loading of optimized wing shapes for eight aerofoil modes applied locally ( 82 variables case) and globally (10 variable case) behaviour of the modes is preserved through to the optimization and lead to monotonic improvement in objective with increase in dimensionality. No adverse optimization performance of these modes were found when structural response was included, so this approach is suitable for such optimization problems.

Further, utilising the flexibility of the approach, global sectional optimization was performed and it was shown that for an equivalent set of modes to localised deformation (10 design variables for global deformations and 82 for local), that there was only a $4 \%$ difference in the final optimization result. The global deformations therefore show great promise when it comes to being utilised with a global optimization algorithm, where low dimensionality is a key since cost scales quadratically with design variables for these algorithms. This opens up the possibility of performing high-fidelity aeroelastic wing shape optimization with global optimization algorithms, which could have particularly use when considering problems that change planform as well as section. 

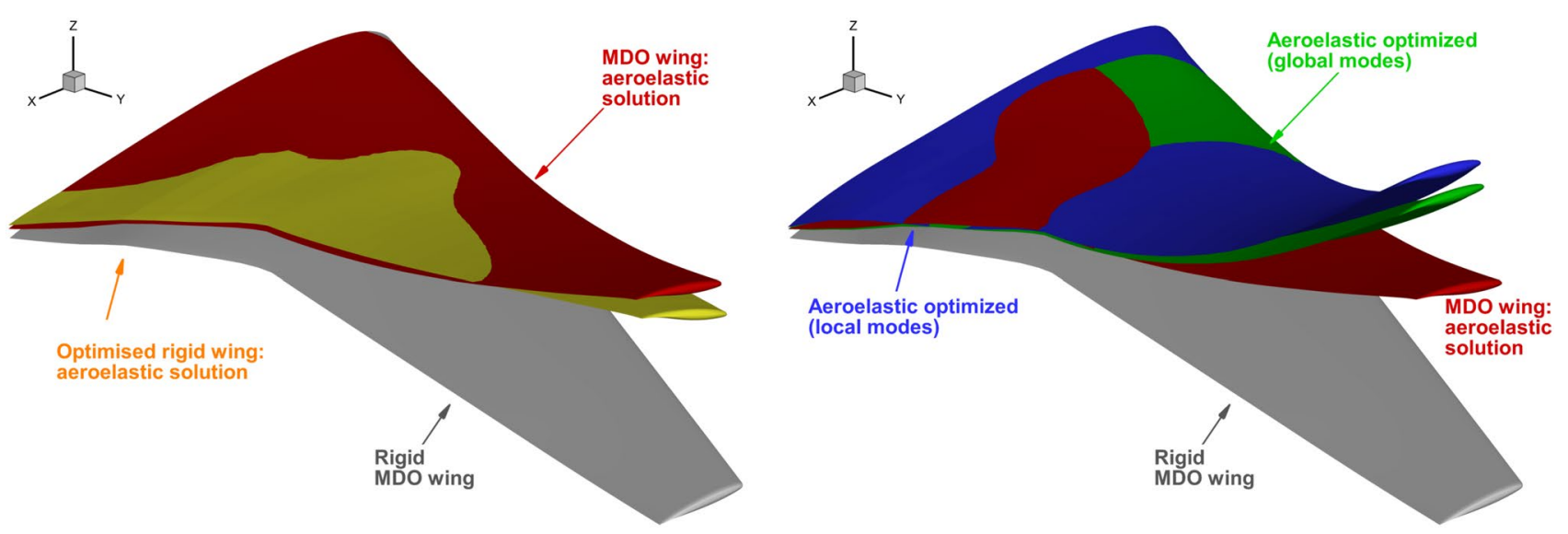

Fig. 18 Iso view of various wings (optimised cases are based on 8 SVD modes)

Acknowledgements The authors kindly acknowledge the support of the EPSRC-supported University of Bristol EPSRC Doctoral Training Grant, which was used to fund the precursor of this work, without which, this research would not have been possible. This work was carried out using the Blue Crystal computational facilities of the Advanced Computing Research Centre, University of Bristol-http:// www.bris.ac.uk/acrc/.

\section{Declarations}

Conflict of interest The authors declare that they have no conflict of interest.

Replication of Results All meshes required to replicate the results are available through the University of Bristol Research Data Repository https://data.bris.ac.uk/data/.

Open Access This article is licensed under a Creative Commons Attribution 4.0 International License, which permits use, sharing, adaptation, distribution and reproduction in any medium or format, as long as you give appropriate credit to the original author(s) and the source, provide a link to the Creative Commons licence, and indicate if changes were made. The images or other third party material in this article are included in the article's Creative Commons licence, unless indicated otherwise in a credit line to the material. If material is not included in the article's Creative Commons licence and your intended use is not permitted by statutory regulation or exceeds the permitted use, you will need to obtain permission directly from the copyright holder. To view a copy of this licence, visit http://creativecommons.org/licenses/by/4.0/.

\section{References}

Allen CB (2001) Multigrid acceleration of an upwind Euler method for hovering rotor flows. Aeronaut J 105(1051):517-524. https:// doi.org/10.1017/S0001924000017954

Allen CB (2002) Multigrid convergence of inviscid fixed- and rotarywing flows. Int J Numer Methods Fluids 39(2):121-140. https:// doi.org/10.1002/fld.282
Allen CB (2006) Parallel simulation of unsteady hovering rotor wakes. Int J Numer Methods Eng 68(6):632-649. https://doi.org/10.1002/ nme. 1723

Allen CB (2008) Towards automatic structured multiblock mesh generation using improved transfinite interpolation. Int J Numer Methods Eng 74(5):697-733. https://doi.org/10.1002/nme.2170

Allen CB, Rendall TCS (2013) Computational-fluid-dynamics-based optimisation of hovering rotors using radial basis functions for shape parameterisation and mesh deformation. Optim Eng 14:97118. https://doi.org/10.1007/s11081-011-9179-6

Allen CB, Poole DJ, Rendall TCS (2018) Wing aerodynamic optimization using efficient mathematically-extracted modal design variables. Optim Eng 19(2):453-477. https://doi.org/10.1007/ s11081-018-9376-7

Allwright S (1997) Reference aircraft performance and primary sensitivities. Tech. Rep., Technical report D.3.12.R, MDO/TR/BAE/ SA970530/1

Bonnans JF, Panier ER, Tits AL, Zhou JL (1992) Avoiding the maratos effect by means of a nonmonotone line search ii. Inequality constrained problems-feasible iterates. SIAM J Numer Anal 29(4):1187-1202. https://doi.org/10.1137/0729072

Chernukhin O, Zingg DW (2013) Multimodality and global optimization in aerodynamic design. AIAA J 51(6):1342-1354. https://doi.org/10.2514/1.J051835

Destarac D, Carrier G, Anderson GR, Nadarajah S, Poole DJ, Vassberg JC, Zingg DW (2018) Example of a pitfall in aerodynamic shape optimization. AIAA J 56(4):1532-1540. https://doi.org/ 10.2514/1.J056128

Eckart C, Young G (1936) The approximation of one matrix by another of lower rank. Psychometrika 1(3):211-218. https:// doi.org/10.1007/BF02288367

Geuzaine P, Brown G, Harris C, Farhat C (2003) Aeroelastic dynamic analysis of a full $\mathrm{f}-16$ configuration for various flight conditions. AIAA J 41(3):363-371. https://doi.org/10.2514/2. 1975

Ghoman SS, Sarhaddi D, Chen PC, Wang Z, Kapania RK (2012) A hybird optimization strategy using design-space evolution and POD-based order reduction. In: 12th AIAA aviation technology, integration and operations (ATIO) conference and 14th AIAA/ ISSMO multidisciplinary analysis optimization conference, Indianapolis, https://doi.org/10.2514/6.2012-5631, AIAA Paper 2012-5631 
Grippo L, Lampariello F, Lucidi S (1986) A nonmonotone line search technique for Newton's method. SIAM J Numer Anal 23(4):707716. https://doi.org/10.1137/0723046

Haase D, Selmin V, Winzell B (2002) Progress in computational flowstructure interaction, Springer, chap Notes on Numerical Fluid Mechanics and Multidisciplinary design

Hicks RM, Henne PA (1978) Wing design by numerical optimization. J Aircraft 15(7):407-412. https://doi.org/10.2514/6.1977-1247

Hübner B, Walhorn E, Dinkler D (2004) A monolithic approach to fluid-structure interaction using space-time finite elements. Comput Methods Appl Mech Eng 193(23-26):2087-2104. https://doi. org/10.1016/j.cma.2004.01.024

Jameson A (1988) Aerodynamic design via control theory. J Sci Comput 3(3):233-260. https://doi.org/10.1007/BF01061285

Jameson A, Schmidt W, Turkel E (1981) Numerical solution of the Euler equations by finite volume methods using Runge Kutta time stepping schemes. In: 14th Fluid and Plasma Dynamics Conference, Palo Alto, CA, https://doi.org/10.2514/6.1981-1259, AIAA Paper 1981-1259

Kedward LJ, Allen CB, Rendall TCS (2020) Gradient-limiting shape control for efficient aerodynamic optimization. AIAA J 58(9):3748-3764. https://doi.org/10.2514/1.J058977

Kedward LJ, Allen CB, Rendall TCS, Poole DJ (2020b) Generic modal design variables for aerodynamic shape optimisation. In: AIAA AVIATION Forum, https://doi.org/10.2514/6.2020-2707, AIAA Paper 2020-2707

Kenway GKW, Martins JRRA (2014) Multi-point high-fidelity aerostructural optimization of a transport aircraft configuration. J Aircraft 51(1):144-160. https://doi.org/10.2514/1.C032150

Kenway GKW, Kennedy GJ, Martins JRRA (2014) Scalable parallel approach for high-fidelity steady-state aeroelastic analysis and adjoint derivative computations. AIAA J 52(5):935-951. https:// doi.org/10.2514/1.J052255

Keye S, Mavriplis D (2018) Summary of case 5 from sixth drag prediction workshop: Coupled aerostructural simulation. J Aircraft 55(4):1380-1387. https://doi.org/10.2514/1.C034427

Lyu Z, Xu Z, Martins JRRA (2014) Benchmarking optimization algorithms for wing aerodynamic design optimization. In: Eighth International Conference on Computational Fluid Dynamics (ICCFD8), Chegdu, iCCFD8-2014-0203

Maratos N (1978) Exact penalty function algorithms for finite dimensional and optimization problems. $\mathrm{PhD}$ thesis, Imperial College

Martins JRRA, Lambe AB (2013) Multidisciplinary design optimization: a survey of architectures. AIAA J 51(9):2049-2075. https:// doi.org/10.2514/1.J051895

Martins JRRA, Alonso JJ, Reuther JJ (2005) A coupled-adjoint sensitivity analysis method for high-fidelity aero-structural design. Optim Eng 6(1):33-62. https://doi.org/10.1023/B:OPTE.00000 48536.47956.62

Masters DA, Taylor NJ, Rendall TCS, Allen CB, Poole DJ (2017) Geometric comparison of aerofoil shape parameterization methods. AIAA J 55(5):1575-1589. https://doi.org/10.2514/1.J0549 43

Maute K, Nikbay M, Farhat C (2001) Coupled analytical sensitivity analysis and optimization of three-dimensional nonlinear aeroelastic systems. AIAA J 39(11):2051-2061. https://doi.org/ $10.2514 / 2.1227$

Mavriplis D, Fabiano E, Anderson E (2017) Recent advances in high-fidelity multidisciplinary adjoint-based optimization with the nsu3d flow solver. In: 55th AIAA Aerospace Sciences Meeting, Grapevine, TX, https://doi.org/10.2514/6.2017-1669, AIAA Paper 2017-1669
Mayne DQ, Polack E (1982) A surperlinearly convergent algorithm for constrained optimization problems. Math Program Stud 4:45-61. https://doi.org/10.1007/BFb0120947

Michler C, Hulshoff SJ, van Brummelen EH, de Borst R (2004) A monolithic approach to fluid-structure interaction. Comput Fluids 33(5-6):839-848. https://doi.org/10.1016/j.compfluid. 2003.06.006

Morris AM, Allen CB, Rendall TCS (2008) CFD-based optimization of aerofoils using radial basis functions for domain element parameterization and mesh deformation. Int J Numer Methods Fluids 58(8):827-860. https://doi.org/10.1002/fld.1769

Newmark NM (1959) A method of computation for structural dynamics. J Eng Mech Div 85(3):67-94

Panier ER, Tits AL (1991) Avoiding the Maratos effect by means of a nonmonotone line search i. General constrained problems. SIAM J Numer Anal 28(4):1183-1195. https://doi.org/10.1137/0728063

Poole DJ, Allen CB, Rendall TCS (2015) Metric-based mathematical derivation of efficient airfoil design variables. AIAA J 53(5):1349-1361. https://doi.org/10.2514/1.J053427

Poole DJ, Allen CB, Rendall TCS (2015b) Optimal domain element shapes for free-form aerodynamic shape control. In: 53rd AIAA Aerospace Sciences Meeting, Kissimmee, FL, https://doi.org/10. 2514/6.2015-0762, aIAA Paper 2015-0762

Poole DJ, Allen CB, Rendall TCS (2017) High-fidelity aerodynamic shape optimization using efficient orthogonal modal design variables with a constrained global optimizer. Comput Fluids 143:115. https://doi.org/10.1016/j.compfluid.2016.11.002

Poole DJ, Allen CB, Rendall TCS (2018) Comparison of point design and range-based objectives for transonic aerofoil optimization. AIAA J 56(8):3240-3256. https://doi.org/10.2514/1.J056627

Poole DJ, Allen CB, Rendall TCS (2018) Global optimization of wing aerodynamic optimization case exhibiting multimodality. J Aircraft 55(4):1576-1591. https://doi.org/10.2514/1.C034718

Qin N, Vavalle A, Le Moigne A, Laban M, Hackett K, Weinerfelt P (2004) Aerodynamic considerations of blended wing body aircraft. Progress Aerospace Sci 40(6):321-343. https://doi.org/10. 1016/j.paerosci.2004.08.001

Rendall TCS, Allen CB (2008) Unified fluid-structure interpolation and mesh motion using radial basis functions. Int. J Numer Methods Eng 74(10):1519-1559. https://doi.org/10.1002/nme.2219

Rendall TCS, Allen CB (2010) Reduced surface point selection options efficient mesh deformation using radial basis functions. J Comput Phys 229(8):2810-2820. https://doi.org/10.1016/j.jcp.2009.12.006

Sobieszczanski-Sobieski J, Haftka RT (1997) Multidisciplinary aerospace design optimization: survey of recent developments. Struct Optim 14(1):1-23. https://doi.org/10.1007/BF01197554

Streuber GM, Zingg DW (2021) On the risk of local optima in aerodynamic shape optimization. AIAA J 59(1):75-87. https://doi.org/ 10.2514/1.J059826

Taylor NV, Allen CB, Gaitonde A, Jones DP (2004) A structure-coupled CFD method for time-marching flutter analysis. Aeronaut $\mathrm{J}$ 108(1086):389-401. https://doi.org/10.1017/S0001924000000208

Toal DJJ, Bressloff NW, Keane AJ, Holden CME (2010) Geometric filtration using proper orthogonal decomposition for aerodynamic design optimization. AIAA J 48(5):916-928. https://doi.org/10. $2514 / 1.41420$

Wendland H (2005) Scattered data approximation, 1st edn. Cambridge University Press, Cambridge

Woodgate MA, Badcock KJ (2005) Aeroelastic calculations for the hawk aircraft using the Euler equations. J Aircraft 42(4):1005-1011

Yu Y, Lyu Z, Xu Z, Martins JRRA (2018) On the influence of optimization algorithm and initial design on wing aerodynamic shape 
optimization. Aerospace Sci Technol 75:183-199. https://doi.org/ 10.1016/j.ast.2018.01.016

Zhang ZJ, Khosravi S, Zingg DW (2017) High-fidelity aerostructural optimization with integrated geometry parameterization and mesh movement. Struct Multidisc Optim 55:1217-1235. https://doi.org/ 10.1007/s00158-016-1562-7

Zhou JL, Tits AL, Lawrence CT (1997) Users guide for FSQP version 3.7: a fortran code for solving optimization programs, possibly minimax, with general inequality constraints and linear equality constraints, generating feasible iterates. Tech. Rep., Institute for Systems Research, University of Maryland, sRC-TR-92-107r5

Publisher's Note Springer Nature remains neutral with regard to jurisdictional claims in published maps and institutional affiliations. 